\title{
Anisotropic D3-D5 black holes with unquenched flavors
}

\author{
José Manuel Penín, ${ }^{a, b}$ Alfonso V. Ramallo ${ }^{a, b}$ and Dimitrios Zoakos ${ }^{c}$ \\ ${ }^{a}$ Departamento de Física de Partículas, Universidade de Santiago de Compostela, \\ Xoaquín Díaz de Rábago S/N, E-15782 Santiago de Compostela, Spain \\ ${ }^{b}$ Instituto Galego de Física de Altas Enerxías (IGFAE), \\ Xoaquín Díaz de Rábago S/N, E-15782 Santiago de Compostela, Spain \\ ${ }^{c}$ Centro de Física do Porto, Universidade do Porto, \\ Rua do Campo Alegre 687, 4169-007 Porto, Portugal \\ E-mail: jmanpen@gmail.com, alfonso@fpaxp1.usc.es, zoakos@gmail.com
}

ABSTRACT: We construct a black hole geometry generated by the intersection of $N_{c}$ color D3- branes and $N_{f}$ flavor D5-branes along a $2+1$ dimensional subspace. Working in the Veneziano limit in which $N_{f}$ is large and distributing homogeneously the D5-branes in the internal space, we calculate the solution of the equations of motion of supergravity plus sources which includes the backreaction of the flavor branes. The solution is analytic and dual to a $2+1$ dimensional defect in a $3+1$ dimensional gauge theory, with $N_{f}$ massless hypermultiplets living in the defect. The smeared background we obtain can be regarded as the holographic realization of a multilayered system. We study the thermodynamics of the resulting spatially anisotropic geometry and compute the first and second order transport coefficients for perturbations propagating along the defect. We find that, in our system, the dynamics of excitations within a layer can be described by a stack of effective D2-branes.

Keywords: AdS-CFT Correspondence, Brane Dynamics in Gauge Theories, D-branes, Gauge-gravity correspondence

ARXIV EPRINT: 1710.00548 


\section{Contents}

1 Introduction $\quad 2$

2 The D3-D5 black hole 4

3 Thermodynamics of the black hole $\quad 7$

$\begin{array}{lll}3.1 & \text { Stress-energy tensor } & 12\end{array}$

4 Effective action in $4 d \quad 14$

$\begin{array}{lll}4.1 & \text { Stress-energy tensor } & 17\end{array}$

5 Effective action in 5d $\quad 18$

$\begin{array}{llr}5.1 & \text { Stress-energy tensor } & 19\end{array}$

$\begin{array}{ll}5.2 \text { Holographic dictionary } & 20\end{array}$

6 Fluctuations and hydrodynamics $\quad 21$

6.1 The shear channel 23

$\begin{array}{ll}6.2 & \text { The sound channel } \\ \end{array}$

$\begin{array}{lll}7 & \text { Summary and conclusions } & 31\end{array}$

A Details of the background 33

B Wilson loops and entanglement entropies $\quad 34$

B.1 Quark-antiquark potentials 34

B.1.1 Intra-layer potential 35

B.1.2 Inter-layer potential 36

B.2 Entanglement entropy 37

$\begin{array}{ll}\text { B.2.1 Parallel slab } & 37\end{array}$

$\begin{array}{lll}\text { B.2.2 Transverse slab } & 39\end{array}$

C More on the reduced equations $\quad 40$

C.1 4d reduction 40

$\begin{array}{ll}\text { C.2 } 5 \text { d reduction } & 42\end{array}$

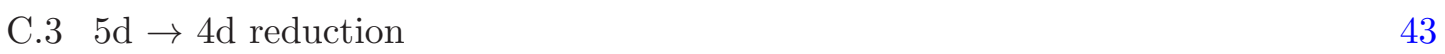

D Hydrodynamic fluctuations $\quad 44$

D.1 Shear channel 44

D.2 Sound channel 45 


\section{Introduction}

The holographic AdS/CFT correspondence [1] has become a useful and powerful tool to study quantum field theories in the strongly coupled regime (see [2-4] for reviews). Even if most of the models studied in the holographic framework are very different from the systems found in the phenomenology, many of the results obtained using them are believed to be universal. To test the universality of these holographic results one should be able to extend the holographic analysis to models including features present in real life systems.

In this paper we construct a model which allows to explore the extension of the AdS/CFT correspondence in two directions. First of all, we add dynamical flavors, i.e., fields transforming in the fundamental representation of the gauge group. Moreover, our model is dual to a four-dimensional system which is spatially anisotropic since one of the spatial field theory directions of the metric is distinguished with respect to the other two. The corresponding geometry is a black hole, i.e., it has an event horizon, and is based on the D3-D5 brane intersection of type IIB supergravity. The D3-branes are the color branes which, in the absence of D5-branes, generate the $A d S_{5} \times S^{5}$ geometry dual to $\operatorname{SU}\left(N_{c}\right)$ $\mathcal{N}=4$ super Yang-Mills in $3+1$-dimensions. The D5-branes are the flavor branes [5] and are arranged in such a way that they create a $(2+1)$-dimensional, codimension one, defect on the worldvolume of the D3-branes.

The field theory dual of this D3-D5 setup is well known. It was determined some time ago in $[6]$ (see also $[7,8]$ ). It consists of a supersymmetric defect theory with $(2+1)$ dimensional matter hypermultiplets coupled to a $(3+1)$-dimensional bulk theory. In the past this D3-D5 setup was extensively studied in the approximation in which the D5-branes are considered as probes in the D3-brane geometry (see, for example, [9-16]). This is the socalled quenched approximation, which corresponds, in the field theory side, to neglecting the quark dynamical effects due to quark loops. This probe brane approach is a good approximation when the number of flavors $N_{f}$ is much smaller than the number of colors $N_{c}$.

In this paper we analyze this D3-D5 brane configuration beyond the quenched approximation. To find gravity duals to unquenched flavor one has to solve the equations of motion of supergravity in the presence of D-brane sources. These sources have Dirac $\delta$-functions and the corresponding Einstein equations are PDE's which are extremely difficult to solve. To overcome this difficulty we follow the proposal of [17] and consider a continuous distribution of D5-brane sources in such a way that there are no $\delta$-functions anymore in our equations of motion. This approach is accurate only when the number of flavors $N_{f}$ is large. Actually, it corresponds to the so-called Veneziano limit, in which both $N_{c}$ and $N_{f}$ are large and their ratio $N_{c} / N_{f}$ is fixed [18]. This smearing approach has been successfully applied to obtain several geometries dual to flavored systems (see [19] for a review and references). In many cases one gets analytic solutions at the price of modifying the $R$-symmetry of the model (due to the average over different orientations of the flavor branes) and changing the flavor group from $\mathrm{U}\left(N_{f}\right)$ to $\mathrm{U}(1)^{N_{f}}$ (the smeared flavor branes are not coincident).

Most of the smeared flavored geometries found in the literature preserve some amount of supersymmetry. Indeed, in these models the preservation of supersymmetry is a crucial guide to find the deformation induced by the flavor branes. However, there are other solu- 
tions which are not supersymmetric and correspond to systems at finite temperature and/or finite baryon density (see [20-24]). For the D3-D5 system we are interested in, the smeared supersymmetric solution has been obtained in [25]. In the case of massless quarks the solution is completely analytic and displays a Lifshitz-like anisotropic scaling symmetry. In this paper we find the non-zero temperature generalization of this scaling background. It turns out that adding an event horizon to the geometry of [25] is straightforward and amounts to adding a blackening factor to the metric. This blackening factor has a non-standard power dependence on the radial coordinate due to the spatial anisotropy of the geometry.

In our background the D5-branes are homogeneously distributed along the internal directions, as well as across the cartesian direction transverse to the defect. Therefore, our gravitational solution should be regarded as the holographic dual of a multilayered system. The different layers are created by the stack of flavor D5-branes distributed in parallel two-dimensional planes inside the three-dimensional space. The resulting system has one distinguished direction and thus it is clearly anisotropic. We want to explore its properties for observables living in a single layer and also for those connecting two different layers. We will find that, non-trivially, the intra-layer dynamics is the same as that of a stack of effective D2-branes, which means that strongly coupled 2+1 super Yang-Mills can be used to describe our system. We will also be able to study some inter-layer properties.

In the condensed matter context it is quite common to have materials with stratified structures containing multiple parallel layers. The possibility of having a holographic topdown model with multiple layers is one of the main motivations for this work. It is worth recalling in this respect that the D3-D5 brane intersection has been used to model the quantum Hall effect and as a holographic model of graphene [13-15].

We will start our analysis by studying the thermodynamics of the D3-D5 black hole and by computing by different methods the VEV of the stress-energy tensor of the dual theory. This analysis will serve us to characterize the anisotropy of the system from the holographic perspective. There is an extensive literature on anisotropic holography. In a by no means exhaustive list, let us mention the articles [26-33], where other backgrounds dual to anisotropic theories have been obtained (some of these geometries are also generated by the backreaction of branes). We will also be able to compute the transport coefficients up to second order for perturbations that propagate along the $(2+1)$-dimensional intersection of the D3- and D5-branes. We will find that these transport coefficients are the same as those of a D2-brane, a result which is not expected a priori.

It is interesting to recall that localized supergravity solutions for the D3-D5 system have already been found in $[34,35]$. These solutions contain cycles with fluxes which can be interpreted as the location of the D5-branes. These D5-branes do not have open string degrees of freedom. This is in contrast to our approach, where the flavor branes are dynamical sources. By smearing these sources we get simpler supersymmetric solutions, which can be easily generalized to construct a black hole.

The organization of the rest of this paper is the following. In section 2 we present our black hole background, whose thermodynamic properties are analyzed in section 3. Besides its temperature and entropy, we obtain the chemical potential associated to the D5-brane charge. This allows us to obtain the Helmhotz and Gibbs free energies and find the speed 
of sound in the directions parallel and orthogonal to the defect. We will check these results by computing the VEV of the stress-energy tensor from the regularized Brown-York tensor of the gravity theory.

In section 4 we obtain an effective gravitational action for our problem in fourdimensions, which we renormalize holographically by means of a suitable boundary counterterm constructed from a superpotential. In section 5 we present a five-dimensional gravitational action for our system, which includes a smeared codimension one DBI contribution due to the D5-branes. The regulating boundary term for this action contains a bulk superpotential, as well as a superpotential generated by the flavor branes. We use both the four and five dimensional regulated actions to calculate the VEV of the stress-energy tensor and to confirm the values obtained in the thermodynamic analysis. In section 6 we use the four-dimensional effective action to compute the transport coefficients in the shear and sound channels. Finally, in section 7 we summarize our results and discuss possible extensions of our work. The paper is completed with four appendices with details of the calculations presented in the main text.

\section{The D3-D5 black hole}

In this section we present the brane setup corresponding to our black hole geometry, as well as its metric and forms. More details are provided in appendix A. Our background is based on the following array of D3- and D5-branes:

$$
\begin{array}{lllllllll}
1 & 2 & 3 & 4 & 5 & 6 & 7 & 8 & 9
\end{array}
$$

$$
\begin{aligned}
& \left(N_{c}\right) D 3: \times \times \times \ldots \ldots \\
& \left(N_{f}\right) D 5: \times \times \ldots \times \times \ldots
\end{aligned}
$$

where the $N_{c}$ D3-branes are color branes and the $N_{f}$ D5-branes are flavor branes. As it is clear from (2.1) the D5-branes create a $(2+1)$-dimensional defect in the $(3+1)$-dimensional bulk gauge theory. In general, the directions 4-9 correspond to a Sasaki-Einstein cone, with the D3-branes located at the tip of the cone. For concreteness we will consider here the case in which the D3-branes are in flat space and, therefore, the base of the cone will be just the five-sphere $\mathbb{S}^{5}$.

The ten-dimensional metric of our geometry in Einstein frame has the factorized form:

$$
d s_{10}^{2}=d s_{5}^{2}+d \hat{s}_{5}^{2},
$$

where $d s_{5}^{2}$ is:

$$
d s_{5}^{2}=\frac{r^{2}}{R^{2}}\left[-b\left(d x^{0}\right)^{2}+\left(d x^{1}\right)^{2}+\left(d x^{2}\right)^{2}+e^{-2 \phi}\left(d x^{3}\right)^{2}\right]+R^{2} \frac{d r^{2}}{b r^{2}},
$$

where $R$ is a constant radius and $b=b(r)$ is the blackening factor, given by:

$$
b=1-\left(\frac{r_{h}}{r}\right)^{\frac{10}{3}},
$$


with $r_{h}$ being the horizon radius. The function $\phi$ multiplying the metric (2.3) along the $x^{3}$ direction is the type IIB supergravity dilaton, which is not constant due to the presence of the D5-branes. The running of $\phi$ characterizes the anisotropy introduced by the flavor branes in the $(3+1)$-dimensional gauge theory.

The metric $d \hat{s}_{5}^{2}$ in (2.2) corresponds to the internal part of the 10d geometry. As in the smeared solution of [25] this internal metric is just a deformed $\mathbb{S}^{5}$. This deformation can be easily described when the $\mathbb{S}^{5}$ is represented as a $U(1)$ bundle over $\mathbb{C} \mathbb{P}^{2}$ : the deformation is just a squashing of the $\mathrm{U}(1)$ fiber relative to the $\mathbb{C P}^{2}$ base. Actually, the internal part of our metric is:

$$
d \hat{s}_{5}^{2}=\bar{R}^{2}\left[d s_{\mathbb{C P}^{2}}^{2}+\frac{9}{8}(d \tau+A)^{2}\right]
$$

where $\bar{R}^{2}$ is a constant related to the radius $R$ as:

$$
\bar{R}^{2}=\frac{9}{8} R^{2} .
$$

Our backreacted background is a solution of the equations of motion derived from the total action of the system, which is the sum of the type IIB supergravity action and of the action of the D5-branes:

$$
S=S_{I I B}+S_{\text {branes }}
$$

The action of type IIB supergravity in Einstein frame is:

$$
S_{I I B}=\frac{1}{2 \kappa_{10}^{2}}\left[\int d^{10} x \sqrt{-g}\left(R-\frac{1}{2} \partial_{\mu} \phi \partial^{\mu} \phi\right)-\int\left(\frac{1}{2} e^{\phi} F_{3} \wedge * F_{3}+\frac{1}{4} F_{5} \wedge * F_{5}\right)\right],
$$

while the action of the branes is given by the sum of DBI and WZ terms:

$$
S_{\text {branes }}=-T_{5} \sum_{N_{f}}\left(\int_{\mathcal{M}_{6}} d^{6} \xi e^{\frac{\phi}{2}} \sqrt{-\hat{g}_{6}}-\int_{\mathcal{M}_{6}} \hat{C}_{6}\right),
$$

where $T_{5}$ is the tension of the D5-brane $\left(1 / T_{5}=(2 \pi)^{5} g_{s}\left(\alpha^{\prime}\right)^{3}\right), \hat{g}_{6}$ is the determinant of the induced metric on the worldvolume $\mathcal{M}_{6}$ and $\hat{C}_{(6)}$ is the pullback to $\mathcal{M}_{6}$ of the RR six-form potential of the type IlB theory. In (2.8) we have only included the RR three- and five-forms $F_{3}$ and $F_{5}$, which are the only non-trivial ones for our D3-D5 geometry.

The stack of color D3-branes induces a self-dual RR five-form $F_{5}$ of the type:

$$
F_{5}=K(r)(1+*) d^{4} x \wedge d r
$$

where $K=K(r)$ is a function of the radial variable whose explicit expression can be found in appendix A (eq. (A.9)). Moreover, the $N_{f}$ flavor D5-branes act as a source of the RR three-form $F_{3}$ through the WZ term of the action (2.9).

In the smearing approach, valid when $N_{f}$ is large, we substitute the discrete distribution of flavor branes by a continuous distribution with the appropriate normalization, in such a way that the smearing amounts to performing the substitution:

$$
\sum^{N_{f}} \int_{\mathcal{M}_{6}} \hat{C}_{(6)} \Longrightarrow \int_{\mathcal{M}_{10}} \Xi \wedge C_{(6)},
$$


where $\Xi$ is a four-form (the so-called smearing form), with components along the directions orthogonal to the worldvolume of the flavor branes, which characterizes the charge distribution of the flavor branes. As shown in [25] this WZ coupling induces the following violation of Bianchi identity of $F_{(3)}$ :

$$
d F_{3}=2 \kappa_{10}^{2} T_{5} \Xi
$$

The detailed form of $F_{3}$ and $\Xi$ in terms of differentials of the coordinates is given in appendix A (see (A.6) and (A.7)). It is important to notice that $\Xi$ does not depend on $x^{3}$ (it only depends on $d x^{3}$ ), which means that we are homogeneously distributing our flavor branes in the $x^{3}$ direction and, therefore, we can regard our setup as dual to a multilayer system. Moreover, $\Xi$ is also independent of the radial coordinate $r$, as expected for a charge distribution corresponding to massless quarks. The radii $R$ and $\bar{R}$ depend on the number of color branes $N_{c}$. Indeed, they can be written as:

$$
R^{4}=\frac{256}{1215} Q_{c}, \quad \bar{R}^{4}=\frac{4}{15} Q_{c},
$$

where $Q_{c}$ is proportional to $N_{c}$ and given by:

$$
Q_{c}=\frac{(2 \pi)^{4} g_{s} \alpha^{2} N_{c}}{\operatorname{Vol}\left(\mathcal{M}_{5}\right)}=16 \pi g_{s} \alpha^{\prime 2} N_{c}
$$

In what follows we will take $g_{s}=\alpha^{\prime}=1$. Moreover, $F_{3}$ and the dilaton $\phi$ depend on the quantity $Q_{f} \sim N_{f}$, as shown in (A.6) and (A.8). The precise relation between $Q_{f}$ and $N_{f}$ is written in (A.10). It is important to point out that our solution is not analytic in $N_{f}$, which means that we cannot take the unflavored limit $N_{f}=0$ and recover the isotropic $A d S_{5} \times \mathbb{S}^{5}$ background. $^{1}$

When $r_{h}=0$ (and $b=1$ ) our solution is supersymmetric, as shown in [25], and can be found by solving a set of first-order BPS equations. This supersymmetric solution is invariant under a set of Lifshitz-like anisotropic scale transformations in which the $x^{3}$ coordinate transforms with an anomalous exponent $z=3$ (see [25] for further details about this scaling symmetry).

In order to explore the physical consequences of the anisotropy of our background, we have computed in appendix B the potential energy, at zero temperature, for a quarkantiquark pair, following the holographic prescription of refs. [36, 37]. We have considered the cases in which the charges are in the same layer (i.e., when they have the same value of $x^{3}$ ) and when they are separated along $x^{3}$. Let us summarize here the results. The intra-layer potential takes the form:

$$
V_{q \bar{q}} \sim \frac{N_{c}^{\frac{2}{3}}}{N_{f}^{\frac{1}{3}}} \frac{1}{d_{\|}^{\frac{4}{3}}},
$$

\footnotetext{
${ }^{1}$ One can take this $N_{f}=0$ limit in the equations of motion but not in their particular solution corresponding to our background.
} 
where $d_{\|}$is the $q \bar{q}$ distance in the $x^{1} x^{2}$ plane. Moreover, for charges with the same values of $\left(x^{1}, x^{2}\right)$ and separated a distance $d_{\perp}$ along the coordinate $x^{3}$, we obtain:

$$
V_{q \bar{q}} \sim \frac{N_{c}^{2}}{N_{f}^{3}} \frac{1}{d_{\perp}^{4}} .
$$

The different behaviors (2.15) and (2.16) gives us a measure of the effects of the anisotropy on physical observables. Another effect of this anisotropy is encoded in the entanglement entropies for slab regions and their complements at zero temperature. For a slab with a finite width $l_{\|}$in the plane, the entanglement entropy behaves as (see appendix B for details):

$$
S_{\|} \sim \frac{N_{f}^{\frac{2}{3}} N_{c}^{\frac{5}{3}}}{l_{\|}^{\frac{4}{3}}},
$$

whereas if the slab has a finite width $l_{\perp}$ along $x^{3}$ we get:

$$
S_{\perp} \sim \frac{N_{c}^{4}}{N_{f}^{4}} \frac{1}{l_{\perp}^{6}}
$$

Eqs. (2.17) and (2.18) contain information about the quantum correlations of the model. In particular, the dependence of the entropies on the length determines the critical behavior of the mutual information. Interestingly, $S_{\|}$depends on $N_{c}$ and $l_{\|}$as in the case of a D2-brane. We will find several times in this paper this equivalence of the intra-layer physics with the one corresponding to an effective D2-brane.

When $r_{h} \neq 0$ our solution has a horizon and becomes a black hole with a non-zero temperature. In this case one can show that it solves the Einstein equations with sources that follow from the action (2.7). In particular the DBI term of (2.9) contributes to the energy-momentun tensor and, as already mentioned, the WZ term induces a violation of the Bianchi identity of $F_{3}$. In the next section we explore the thermodynamic properties of this black hole.

\section{Thermodynamics of the black hole}

Let us now work out the thermodynamics of the black hole presented in the previous section. First of all, we recall that the temperature $T$ is given by the general formula:

$$
T=\frac{1}{2 \pi}\left[\frac{1}{\sqrt{g_{r r}}} \frac{d}{d r}\left(\sqrt{-g_{x^{0} x^{0}}}\right)\right]_{r=r_{h}},
$$

which leads to the following relation between $T$ and the horizon radius $r_{h}$ :

$$
T=\frac{5 r_{h}}{6 \pi R^{2}} .
$$

Using (2.13) we can recast this relation in terms of $Q_{c}$ as:

$$
r_{h}=\frac{2^{5} \pi}{3^{\frac{3}{2}} 5^{\frac{3}{2}}} Q_{c}^{\frac{1}{2}} T
$$


The entropy density $s$ is given by the Bekenstein-Hawking formula:

$$
s=\frac{2 \pi}{\kappa_{10}^{2}} \frac{A_{8}}{V_{3}},
$$

where $A_{8}$ is the volume at the horizon of the eight-dimensional space orthogonal to $t$ and $r$ and $V_{3}$ is the infinite constant volume of the three-dimensional Minkowski directions. For our black hole geometry we get:

$$
\frac{A_{8}}{V_{3}}=2^{-\frac{11}{3}} 3^{\frac{17}{6}} 5^{-\frac{1}{2}} \pi^{3} Q_{f}^{\frac{2}{3}} Q_{c}^{\frac{1}{2}} r_{h}^{\frac{7}{3}} .
$$

After using (3.3) to relate $r_{h}$ and $T$, we arrive at:

$$
s=\frac{2^{3}}{5^{4} 3^{\frac{2}{3}} \pi^{\frac{2}{3}}} Q_{f}^{\frac{2}{3}} Q_{c}^{\frac{5}{3}} T^{\frac{7}{3}} .
$$

Notice the fractional powers of $Q_{c}$ and $Q_{f}$ in (3.6), which mean that $s$ has a non-standard dependence on $N_{c}$ and $N_{f}$. To explore further this dependence, let us rewrite (3.6) in terms of $N_{c}$ and $N_{f}$. With this purpose we use the relations (2.14) and (A.10), from which we get that the combination appearing in (3.6) is given by:

$$
Q_{f}^{\frac{2}{3}} Q_{c}^{\frac{5}{3}}=\frac{256}{33^{\frac{2}{3}}} \pi^{\frac{7}{3}} N_{f}^{\frac{2}{3}} N_{c}^{\frac{5}{3}},
$$

and the entropy density can be written as:

$$
s=\alpha_{s} N_{f}^{\frac{2}{3}} N_{c}^{\frac{5}{3}} T^{\frac{7}{3}}
$$

where $\alpha_{s}$ is the following numerical coefficient:

$$
\alpha_{s}=\frac{2048}{5625} \frac{\pi^{\frac{5}{3}}}{3^{\frac{1}{3}}} \approx 1.701 .
$$

The ADM energy of the background is given by the standard equation:

$$
E_{A D M}=-\frac{1}{\kappa_{10}^{2}} \sqrt{\left|g_{t t}\right|} \int_{\mathcal{M}_{t, r_{\infty}}} \sqrt{\operatorname{det} g_{8}}\left(K_{T}-K_{0}\right)
$$

where the symbols $K_{T}$ and $K_{0}$ denote the extrinsic curvatures of the eight-dimensional subspace within the nine-dimensional (constant time) space, at finite and zero temperature, respectively. For an arbitrary hypersurface the extrinsic curvature $K$ is given by:

$$
K=\frac{1}{\sqrt{\operatorname{det} g_{9}}} \partial_{\mu}\left(\sqrt{\operatorname{det} g_{9}} n^{\mu}\right),
$$

with $n^{\mu}$ being a normalized vector perpendicular to the surface. For a constant $r$ hypersurface, we have:

$$
n^{\mu}=\frac{1}{\sqrt{g_{r r}}} \delta_{r}^{\mu} .
$$


For our geometry it is straightforward to prove that:

$$
K=\frac{7}{3 R} \sqrt{b},
$$

where $b$ is the blackening factor (2.4). From this result it follows that:

$$
K_{T}=\frac{7}{3 R} \sqrt{1-\left(\frac{r_{h}}{r}\right)^{\frac{10}{3}}}, \quad K_{0}=\frac{7}{3 R},
$$

and thus the difference of the extrinsic curvatures appearing in (3.10) is:

$$
K_{T}-K_{0} \approx-\frac{7}{6 R}\left(\frac{r_{h}}{r}\right)^{\frac{10}{3}}, \quad(r \rightarrow \infty) .
$$

The energy density $\epsilon$ can now be easily computed, with the result:

$$
\epsilon=\frac{E_{A D M}}{V_{3}}=\frac{7}{10} \alpha_{s} N_{f}^{\frac{2}{3}} N_{c}^{\frac{5}{3}} T^{\frac{10}{3}}=\beta_{s} Q_{f}^{\frac{2}{3}} Q_{c}^{\frac{5}{3}} T^{\frac{10}{3}},
$$

where $\alpha_{s}$ is the numerical coefficient (3.9) and we have introduced a new numerical factor $\beta_{s}$, given by:

$$
\beta_{s}=\frac{28}{3125(3 \pi)^{\frac{2}{3}}} .
$$

Notice that the entropy density (3.6) can be rewritten as:

$$
s=\frac{10}{7} \beta_{s} Q_{f}^{\frac{2}{3}} Q_{c}^{\frac{5}{3}} T^{\frac{7}{3}} .
$$

The free energy density $f$ in the canonical ensemble is defined as:

$$
f=\epsilon-T s .
$$

By using (3.8) and (3.16) we readily obtain:

$$
f=-\frac{3}{10} \alpha_{s} N_{f}^{\frac{2}{3}} N_{c}^{\frac{5}{3}} T^{\frac{10}{3}}=-\frac{3}{7} \beta_{s} Q_{f}^{\frac{2}{3}} Q_{c}^{\frac{5}{3}} T^{\frac{10}{3}} .
$$

To explore the complete thermodynamics of the system it is convenient to consider the situation in which the number of flavor D5-branes can change. In our setup this number of flavor branes is determined by $Q_{f}$. Therefore, we allow $Q_{f}$ to vary and we will introduce the chemical potential $\Phi$, conjugate to $Q_{f}$. The first law of thermodynamics for these variables becomes:

$$
d \epsilon=T d s+\Phi d Q_{f} .
$$

Clearly, the chemical potential $\Phi$ measures the energy cost of introducing additional flavor branes in the system. After performing the Legendre transform as in (3.19), we can write the variation of the free energy $f$ in the canonical ensemble as:

$$
d f=-s d T+\Phi d Q_{f} .
$$


It follows immediately from (3.22) that $s$ and $\Phi$ are given by the following partial derivatives of $f$ :

$$
s=-\left(\frac{\partial f}{\partial T}\right)_{Q_{f}}, \quad \Phi=\left(\frac{\partial f}{\partial Q_{f}}\right)_{T} .
$$

By using (3.20), it is now straightforward to compute the partial derivative of $f$ with respect to $T$ and check the first equation in (3.23). Moreover, by computing the derivative of (3.20) with respect to $Q_{f}$ we obtain the expression of the chemical potential $\Phi$ :

$$
\Phi=-\frac{2}{7} \beta_{s} Q_{f}^{-\frac{1}{3}} Q_{c}^{\frac{5}{3}} T^{\frac{10}{3}} .
$$

The Gibbs free energy, i.e., the thermodynamic potential in the grand canonical ensemble, is defined as:

$$
g=f-\Phi Q_{f}
$$

Plugging (3.20) and (3.24) on the right-hand side of (3.25) we get the value of $g$ for our system:

$$
g=-\frac{1}{7} \beta_{s} Q_{f}^{\frac{2}{3}} Q_{c}^{\frac{5}{3}} T^{\frac{10}{3}} .
$$

As argued in [27] (see also [40]), the two thermodynamic potentials $f$ and $g$ are related to the pressure in the $x^{1} x^{2}$ plane $\left(p_{x y}\right)$ and in the $x^{3}$ direction $\left(p_{z}\right)$ as:

$$
f=-p_{x y}, \quad g=-p_{z} .
$$

To demonstrate these identifications of the free energies with the pressures one should take into account the extensivity of the energy and the anisotropic character of our system (details can be found in [27]). In our system these pressures are thus given by:

$$
p_{x y}=\frac{3}{7} \beta_{s} Q_{f}^{\frac{2}{3}} Q_{c}^{\frac{5}{3}} T^{\frac{10}{3}}=\frac{3}{7} \epsilon, \quad p_{z}=\frac{1}{7} \beta_{s} Q_{f}^{\frac{2}{3}} Q_{c}^{\frac{5}{3}} T^{\frac{10}{3}}=\frac{1}{7} \epsilon .
$$

The speeds of sound along the $x^{1} x^{2}$ and $x^{3}$ are defined as:

$$
v_{x y}^{2}=\left(\frac{\partial p_{x y}}{\partial \epsilon}\right)_{Q_{f}}, \quad v_{z}^{2}=\left(\frac{\partial p_{z}}{\partial \epsilon}\right)_{Q_{f}} .
$$

Using (3.28) we can readily evaluate the derivatives on the right-hand side of (3.29), with the result:

$$
v_{x y}^{2}=\frac{3}{7}, \quad v_{z}^{2}=\frac{1}{7}
$$

to be compared with the value $v_{s}^{2}=1 / 2$ for a $2 \mathrm{~d}$ CFT and $v_{s}^{2}=1 / 3$ for a $3 \mathrm{~d} \mathrm{CFT.}{ }^{2}$

The pressure difference is a manifestation of the anisotropy of the system and is measured by the non-vanishing chemical potential. Actually, it is straightforward to verify that, for our system, one has:

$$
p_{z}-p_{x y}=\Phi Q_{f} .
$$

\footnotetext{
${ }^{2}$ The speed of sound for a Dp-brane is $v_{s}^{2}=\frac{5-p}{9-p}$. Therefore $v_{x y}$ coincides with the speed of sound of a D2-brane.
} 
Moreover, we have the following equation of state:

$$
\epsilon=2 p_{x y}+p_{z} .
$$

By combining (3.31) and (3.32) we can obtain the two pressures as functions of $\epsilon$ and $Q_{f}$ :

$$
p_{x y}=\frac{1}{3} \epsilon-\frac{1}{3} \Phi Q_{f}, \quad p_{z}=\frac{1}{3} \epsilon+\frac{2}{3} \Phi Q_{f} .
$$

It is also easy to relate the different quantities to the entropy:

$$
\epsilon=\frac{7}{10} T s, \quad f=-\frac{3}{10} T s, \quad g=-\frac{1}{10} T s, \quad \Phi Q_{f}=-\frac{1}{5} T s .
$$

From these equations one can show that the following relation holds:

$$
\epsilon=\frac{3}{4} T s+\frac{1}{4} \Phi Q_{f},
$$

as well as the so-called Gibbs-Duhem relations:

$$
\epsilon+p_{x y}=T s, \quad \epsilon+p_{z}=T s+\Phi Q_{f} .
$$

Finally, the heat capacity is:

$$
c_{v}=\partial_{T} \epsilon=\frac{7}{3} \alpha_{s} N_{f}^{\frac{2}{3}} N_{c}^{\frac{5}{3}} T^{\frac{7}{3}}=\frac{10}{3} \beta_{s} Q_{f}^{\frac{2}{3}} Q_{c}^{\frac{5}{3}} T^{\frac{7}{3}} .
$$

To get some insight on the nature of our solution, let us analyze the dependence of the entropy density $s$ on $N_{c}, N_{f}$ and $T$ and let us compare it with some known results for other gravity duals. It follows from (3.18) that $s$ behaves with the temperature as $s \sim T^{\frac{7}{3}}$. For a Dp-brane background $s \sim T^{\frac{9-p}{5-p}}$ [41]. Taking $p=2$ in this last formula we obtain the same behavior as in (3.18). This is an indication that our geometry is related to the one generated by D2-branes. Actually, if we define $\lambda$ as:

$$
\lambda=\frac{N_{c}}{N_{f}^{2}},
$$

then the entropy density (3.18) can be written as:

$$
s \sim N_{c}^{2} \lambda^{-\frac{1}{3}} T^{\frac{7}{3}},
$$

which is exactly the form of the entropy of a D2-brane black hole if $\lambda$ is interpreted as a 't Hooft coupling [41]. ${ }^{3}$ In the case of a stack of $N_{c}$ D2-branes, realizing $2+1$ dimensional super Yang-Mills, the 't Hooft coupling is $\lambda=g_{Y M}^{2} N_{c}\left(\lambda=N_{c}\right.$ in our units). Our result suggests that, in our flavored system, the relevant scaling of the coupling with $N_{c}$ and $N_{f}$ is the one written in (3.38). Notice that having a ratio of the numbers of color and flavors as parameter is very natural in a limit of the Veneziano type. Notice also [42]

\footnotetext{
${ }^{3}$ Equivalently, if we define the temperature-dependent effective dimensionless coupling as $\lambda_{\text {eff }}(T)=\lambda / T$, the entropy density (3.39) can be written as $s \sim N_{c}^{2}\left[\lambda_{\text {eff }}(T)\right]^{-\frac{1}{3}} T^{2}$. We are grateful to Javier Tarrío for suggesting this interpretation of our entropy formula.
} 
that the dimensionless parameter controlling the backreaction of the flavor D5-branes is $\kappa_{10}^{2} N_{f} T_{D 5} R^{-2} \sim N_{f} / \sqrt{N_{c}}$. In this parameter $N_{c}$ and $N_{f}$ scale precisely as in (3.38).

The matching we found of the entropy with the one corresponding to a D2-brane is an indication that the dynamics in the $x^{1} x^{2}$ plane (at least its deviation from conformality) is governed by a $2+1$ dimensional super Yang-Mills theory in the strongly coupled regime. The value of the speed of sound $v_{x y}$ found above points in the same direction. In section 6 we will confirm this fact by computing the hydrodynamic transport coefficients for perturbations propagating in the $x^{1} x^{2}$ plane. Actually, there is a direct way to relate our setup to a system of D2-branes. Indeed, by performing a T-duality transformation along the $x^{3}$ direction we can convert our D3-D5 solution into a D2-D6 geometry, in which the D2's are the color branes and the D6's are the flavor branes. In this D2-D6 solution the $x^{3}$ direction is now a distinguished coordinate transverse to the color branes. The corresponding ten-dimensional metric of type IIA supergravity in the Einstein frame takes the form:

$$
\begin{aligned}
d s_{I I A}^{2}=\left(\frac{4 Q_{f}}{3}\right)^{\frac{1}{4}} \frac{r^{\frac{9}{4}}}{R^{\frac{5}{2}}}[ & -b\left(d x^{0}\right)^{2}+\left(d x^{1}\right)^{2}+\left(d x^{2}\right)^{2}+\frac{R^{4}}{r^{4}} \frac{d r^{2}}{b}+ \\
& \left.+\frac{9}{8} \frac{R^{4}}{r^{2}}\left(\frac{\left(d \bar{x}^{3}\right)^{2}}{r^{\frac{4}{3}}}+d s_{\mathbb{C P}^{2}}^{2}+\frac{9}{8}(d \tau+A)^{2}\right)\right],
\end{aligned}
$$

where $b=b(r)$ is the blackening factor (2.4) and the coordinate $\bar{x}^{3}$ is related to the original cartesian coordinate $x^{3}$ by the following rescaling:

$$
\bar{x}^{3}=\left(\frac{4 \sqrt{2}}{9 Q_{f}}\right)^{\frac{1}{3}} x^{3} .
$$

Notice that the D2-branes in this D2-D6 solution are smeared in $x^{3}$, since none of the functions of the metric depends on this coordinate.

This type IIA background is also endowed with a running dilaton $\phi_{I I A}$, as well as RR two- and four-forms, given by:

$$
\begin{aligned}
e^{2 \phi_{I I A}} & =\left(\frac{3}{4 Q_{f}}\right)^{\frac{7}{3}} R^{2} r^{\frac{1}{3}} \\
F_{2} & =Q_{f} \operatorname{Im}\left(\hat{\Omega}_{2}\right) \\
F_{4} & =\frac{20}{3}\left(\frac{2 Q_{f}^{2}}{9}\right)^{\frac{1}{3}} \frac{r^{\frac{7}{3}}}{R^{4}} d r \wedge d x^{0} \wedge d x^{1} \wedge d x^{2} .
\end{aligned}
$$

\subsection{Stress-energy tensor}

The energy density and the pressures of our model can also be obtained by calculating the holographic stress-energy tensor. We will compute this tensor by using several methods and we will check that one gets the same results as those we obtained in the previous subsection by using anisotropic thermodynamics. In this subsection we will compute the VEV of the stress-energy tensor from the Brown-York tensor at the boundary, following the prescription of [43]. In sections 4 and 5 we will dimensionally reduce our ten-dimensional 
theory and will calculate the stress-energy tensor by holographic renormalization, after adding suitable boundary terms to the reduced actions.

The Brown-York tensor of the ten-dimensional gravity theory is:

$$
\tau_{i j}=\frac{1}{\kappa_{10}^{2}}\left(K_{i j}-K \gamma_{i j}\right)
$$

where $\gamma_{i j}$ is the induced metric at a $r=$ constant surface, $K_{i j}$ is the extrinsic curvature of the surface and $K=\gamma^{i j} K_{i j}$. The VEV of the stress-energy tensor of the dual theory is related to the Minkowski components of the Brown-York tensor at the boundary [43]:

$$
\left\langle T^{\mu}{ }_{\nu}\right\rangle=\left.V_{S E} \sqrt{-\gamma_{\mathrm{Min}}} \tau^{\mu}{ }_{\nu}\right|_{\mathrm{reg}, r_{\Lambda} \rightarrow \infty},
$$

where $V_{S E}$ is the volume for the compact 5 d part of the metric, which for the $S^{5}$ is

$$
V_{S E}=\left(\frac{9 \pi}{8}\right)^{3} R^{5}
$$

In (3.44) $\gamma_{\text {Min }}$ is the determinant of the Minkowski part of the induced metric. The righthand side of (3.44) is divergent at the UV boundary. We will give below a precise prescription to eliminate this divergence.

The extrinsic curvature tensor $K_{i j}$ can be obtained from the covariant expression:

$$
K_{i j}=-\frac{1}{2}\left(\nabla_{i} n_{j}+\nabla_{j} n_{i}\right)
$$

where $n_{i}$ are the components of the normal vector to the $r=$ constant surface $\left(n^{i} n_{i}=1\right)$. In a diagonal metric as the one we have in (2.3), the vector $n_{i}$ is given by:

$$
n_{i}=\sqrt{g_{r r}} \delta_{i}^{r} .
$$

Let us now introduce the notation:

$$
\begin{array}{ll}
g_{x^{0} x^{0}} \equiv-k_{1}^{2}=-\frac{r^{2}}{R^{2}} b, & g_{x^{1} x^{1}}=g_{x^{2} x^{2}} \equiv k_{2}^{2}=\frac{r^{2}}{R^{2}}, \\
g_{x^{3} x^{3}} \equiv k_{3}^{2}=\frac{1}{R^{2}}\left(\frac{4 Q_{f}}{3}\right)^{\frac{4}{3}} r^{\frac{2}{3}}, & g_{r r} \equiv k_{r}^{2}=\frac{R^{2}}{r^{2} b},
\end{array}
$$

where we are assuming that the metric is given by (2.3) and (2.5). With these notations, we have:

$$
\sqrt{-\gamma_{\text {Min }}}=k_{1} k_{2}^{2} k_{3}=\frac{1}{R^{4}}\left(\frac{4 Q_{f}}{3}\right)^{\frac{2}{3}} r^{\frac{10}{3}} b^{\frac{1}{2}},
$$

and it is straightforward to compute the components of the extrinsic curvature along the Minkowski directions. The non-vanishing components are:

$$
\begin{aligned}
K_{x^{0} x^{0}} & =\frac{k_{1} k_{1}^{\prime}}{k_{r}}, & K_{x^{1} x^{1}} & =K_{x^{2} x^{2}}=-\frac{k_{2} k_{2}^{\prime}}{k_{r}}, \\
K_{x^{3} x^{3}} & =-\frac{k_{3} k_{3}^{\prime}}{k_{r}}, & K & =-\frac{1}{k_{r}} \partial_{r} \log \left(k_{1} k_{2}^{2} k_{3}\right) .
\end{aligned}
$$


Plugging these results in (3.43) we get explicitly the non-zero components of the BrownYork tensor:

$$
\begin{aligned}
& \tau_{x^{0}}^{x^{0}}=\frac{1}{\kappa_{10}^{2}} \frac{1}{k_{r}} \partial_{r} \log \left(k_{2}^{2} k_{3}\right)=\frac{1}{\kappa_{10}^{2} R} \frac{7}{3} b^{\frac{1}{2}} \\
& \tau_{x^{x^{1}}}^{x^{1}}=\tau_{x^{2}}^{x^{2}}=\frac{1}{2 \kappa_{10}^{2} R} r b^{\frac{1}{2}} \partial_{r} \log \left(r^{\frac{14}{3}} b\right)=\frac{1}{\kappa_{10}^{2} R} \frac{1}{3 b^{\frac{1}{2}}}\left[7-2\left(\frac{r_{h}}{r}\right)^{\frac{10}{3}}\right], \\
& \tau_{x^{x^{3}}}=\frac{1}{2 \kappa_{10}^{2} R} r b^{\frac{1}{2}} \partial_{r} \log \left(r^{6} b\right)=\frac{1}{\kappa_{10}^{2} R} \frac{1}{b^{\frac{1}{2}}}\left[3-\frac{4}{3}\left(\frac{r_{h}}{r}\right)^{\frac{10}{3}}\right] .
\end{aligned}
$$

Let us now specify the regulating procedure we will employ to compute $\left\langle T^{\mu}{ }_{\nu}\right\rangle$. Since we are interested in matching the thermodynamic values found above, it is enough to subtract the zero temperature supersymmetric value, as it was done in [20] for the D3-D7 system. More concretely, we will take $\left\langle T^{\mu}{ }_{\nu}\right\rangle$ to be given by:

$$
\left\langle T_{\nu}^{\mu}\right\rangle=V_{S E} \lim _{r_{\Lambda} \rightarrow \infty}\left[\sqrt{-\gamma_{\mathrm{Min}}} \tau_{\nu}^{\mu}-b^{\frac{1}{2}} \lim _{r_{h} \rightarrow 0}\left(\sqrt{-\gamma_{\mathrm{Min}}} \tau_{\nu}^{\mu}\right)\right]_{r=r_{\Lambda}},
$$

where the $b^{\frac{1}{2}}$ factor is introduced to match the geometries at the cutoff. Using (3.49) and (3.51) we get that the only non-zero components of $\left\langle T^{\mu}{ }_{\nu}\right\rangle$ are:

$$
\left\langle T_{x^{0}}^{x^{0}}\right\rangle=-\epsilon, \quad\left\langle T_{x^{1}}^{x^{1}}\right\rangle=\left\langle T_{x^{2}}^{x^{2}}\right\rangle=\frac{3 \epsilon}{7}, \quad\left\langle T_{x^{3}}^{x^{3}}\right\rangle=\frac{\epsilon}{7},
$$

where $\epsilon$ is the ADM energy density (3.16). Equivalently, we can write the VEV of the stress-energy tensor as:

$$
\left\langle T^{\mu}{ }_{\nu}\right\rangle=\operatorname{diag}\left(-\epsilon, p_{x y}, p_{x y}, p_{z}\right),
$$

where $p_{x y}$ and $p_{z}$ are precisely the values of the pressures found before by introducing the chemical potential.

Notice that the calculation of $p_{x y}$ and $p_{z}$ using the Brown-York tensor depends on the behavior of the geometry as we increase the holographic coordinate $r$ and approach the boundary. On the contrary, the calculation of the pressures based on $\Phi$ is determined by the behavior of the geometry as we vary the flavor charge $Q_{f}$. The agreement of the results found by these two methods is a non-trivial consistency check of our gravity dual.

\section{Effective action in $4 \mathrm{~d}$}

In order to apply the full machinery of the holographic duality to our system it is quite convenient to integrate the action over the internal manifold and convert our problem into a system of low dimensional gravity. There are two possible approaches to carry out this reduction. First of all, we could consider the $x^{3}$ coordinate as internal and reduce the system to a four-dimensional system in the coordinates $\left(t, x^{1}, x^{2}, r\right)$. This is the point of view we will adopt in this section. This approach is very useful to study the dynamics of the system in the $\left(x^{1}, x^{2}\right)$ plane and, indeed, we will use the results of this section in our analysis of the hydrodynamics modes of section 6 . Alternatively, we could include $x^{3}$ in 
our set of reduced coordinates and deal with a five-dimensional anisotropic problem. We will analyze this $5 \mathrm{~d}$ reduction in the next section.

The reduction of our problem to a low dimensional gravity system will allow us to implement a holographic renormalization procedure. We will be able to compute in this framework the VEV of the stress-energy tensor and to confirm the thermodynamic results of section 3.1. Moreover, in section 6 we will study the fluctuations of the $4 \mathrm{~d}$ fields and we will obtain some hydrodynamic coefficients.

Let us consider the following reduction ansatz to four dimensions of the 10d metric:

$$
d s_{10}^{2}=e^{\frac{10}{3} \gamma-\beta} g_{m n} d z^{m} d z^{n}+e^{\frac{10}{3} \gamma+2 \beta}\left(d x^{3}\right)^{2}+e^{-2(\gamma+\lambda)} d s_{\mathbb{C P}^{2}}^{2}+e^{2(4 \lambda-\gamma)}(d \tau+A)^{2},
$$

where $g_{m n}=g_{m n}(z)$ is a $4 \mathrm{~d}$ metric and the scalar fields $\gamma, \lambda$ and $\beta$ depend on the $4 \mathrm{~d}$ coordinates $z^{m}=\left(t, x^{1}, x^{2}, r\right)$. In addition, in the reduced theory we have the dilaton field $\phi=\phi(z)$. The action of this $4 \mathrm{~d}$ gravity theory can be obtained from the one of type IIB supergravity. The details of this calculation are given in appendix C. The expression of this effective action is:

$$
S_{\mathrm{eff}}=\frac{V_{5} V_{x^{3}}}{2 \kappa_{10}^{2}} \int d^{4} z \sqrt{-g_{4}}\left[R_{4}-\frac{40}{3}(\partial \gamma)^{2}-20(\partial \lambda)^{2}-\frac{3}{2}(\partial \beta)^{2}-\frac{1}{2}(\partial \phi)^{2}-V\right],
$$

where $V$ is the following potential for the scalar fields $\phi, \gamma, \lambda$ and $\beta$ :

$$
V=4 e^{\frac{16}{3} \gamma+12 \lambda-\beta}-24 e^{\frac{16}{3} \gamma+2 \lambda-\beta}+Q_{f}^{2} e^{4 \gamma+4 \lambda-3 \beta+\phi}+\frac{Q_{c}^{2}}{2} e^{\frac{40}{3} \gamma-\beta}+6 Q_{f} e^{\frac{14}{3} \gamma-2 \lambda-2 \beta+\frac{\phi}{2}}
$$

In order to write the equations of motion of the reduced theory in a compact form, let us collect the four scalar fields in a single vector $\Psi$ with components:

$$
\Psi=(\phi, \gamma, \lambda, \beta)
$$

Moreover, we define a coefficient $\alpha_{\Psi}$ which takes the following values for the different scalar fields:

$$
\left(\alpha_{\phi}, \alpha_{\gamma}, \alpha_{\lambda}, \alpha_{\beta}\right)=\left(1, \frac{3}{80}, \frac{1}{40}, \frac{1}{3}\right)
$$

Then, Einstein equations can be compactly written in terms of $\Psi$ as:

$$
R_{m n}=\sum_{\Psi} \frac{1}{2 \alpha_{\Psi}} \partial_{m} \Psi \partial_{n} \Psi+\frac{1}{2} g_{m n} V
$$

Moreover, if we define the d'Alembertian of any scalar field $\Psi$ as:

$$
\Psi \equiv \frac{1}{\sqrt{-g_{4}}} \partial_{m}\left(\sqrt{-g_{4}} g^{m n} \partial_{n} \Psi\right),
$$

then, the equations for the scalar fields are:

$$
\Psi=\alpha_{\Psi} \partial_{\Psi} V
$$


Let us now write our black hole solution in terms of the $4 \mathrm{~d}$ variables. The fourdimensional metric takes the diagonal form:

$$
d s_{4}^{2}=-c_{1}^{2}(r) d t^{2}+c_{2}^{2}(r)\left[\left(d x^{1}\right)^{2}+\left(d x^{2}\right)^{2}\right]+c_{3}^{2}(r) d r^{2} .
$$

The actual values of the $c_{i}$ coefficients for our background are:

$$
\begin{aligned}
& c_{1}^{2}(r)=\left(\frac{9}{8}\right)^{3}\left(\frac{4 Q_{f}}{3}\right)^{\frac{2}{3}} R^{2} b(r) r^{\frac{7}{3}}, \\
& c_{2}^{2}(r)=\left(\frac{9}{8}\right)^{3}\left(\frac{4 Q_{f}}{3}\right)^{\frac{2}{3}} R^{2} r^{\frac{7}{3}}=\frac{c_{1}^{2}(r)}{b(r)}, \\
& c_{3}^{2}(r)=\left(\frac{9}{8}\right)^{3}\left(\frac{4 Q_{f}}{3}\right)^{\frac{2}{3}} \frac{R^{6}}{r^{\frac{5}{3}} b(r)}=\frac{R^{4}}{r^{4} b^{2}(r)} c_{1}^{2}(r),
\end{aligned}
$$

where $b(r)$ is the blackening factor defined in (2.4). Moreover, in our geometry the different scalars take the values:

$$
\begin{aligned}
e^{\phi} & =\left(\frac{3}{4 Q_{f}}\right)^{\frac{2}{3}} r^{\frac{2}{3}}, & e^{\gamma} & =\left(\frac{8}{9}\right)^{\frac{3}{5}} \frac{1}{R}, \\
e^{\lambda} & =\left(\frac{9}{8}\right)^{\frac{1}{10}}, & e^{\beta} & =\frac{9}{8}\left(\frac{4 Q_{f}}{3}\right)^{\frac{2}{3}} R^{\frac{2}{3}} r^{\frac{1}{3}} .
\end{aligned}
$$

One can easily verify that these metric and scalar fields solve (4.6) and (4.8).

Let us have a closer look at the $4 d$ metric we obtained. Plugging the $c_{i}(r)$ functions (4.10) into (4.9), we get:

$$
d s_{4}^{2} \sim r^{\frac{7}{3}}\left[-b(r) d t^{2}+(d x)^{1}+\left(d x^{2}\right)^{2}+R^{4} \frac{d r^{2}}{b(r) r^{4}}\right] .
$$

It is easy to check that this metric is equivalent to the one obtained when the $10 \mathrm{~d}$ geometry of the D2-brane is reduced to $4 \mathrm{~d}$ (change to the new radial coordinate $\rho=r^{\frac{3}{2}}$ and compare with the reduced metric written in [44]). Another way of reaching the same conclusion is by noting that under a scale transformation of the type:

$$
t \rightarrow \lambda t, \quad x^{1,2} \rightarrow \lambda x^{1,2}, \quad r \rightarrow r / \lambda
$$

the zero-temperature metric changes homogeneously as:

$$
d s_{4}^{2} \rightarrow \lambda^{-\frac{1}{3}} d s_{4}^{2} .
$$

This behavior corresponds to a hyperscaling violation of the type $d s_{4}^{2} \rightarrow \lambda^{\theta} d s_{4}^{2}$, with hyperscaling violation exponent $\theta=-\frac{1}{3}$ which, as shown in [45], is the $\theta$ exponent corresponding to a D2-brane. However, our 4d theory has more scalars than the reduced theory of a D2-brane and, therefore, even if the metrics are equal, both problems are not equivalent in principle. 


\subsection{Stress-energy tensor}

We now compute the VEV of the stress-energy tensor in this dimensionally reduced gravity theory. First of all we need to renormalize holographically the on-shell action by adding boundary terms. Besides the standard Gibbons-Hawking term, we will add a counterterm constructed with the superpotential for the potential $V$ written in (4.3) [46]. This superpotential will be denoted by $W_{4 d}$ and must satisfy:

$$
V=\frac{1}{2}\left[\frac{3}{80}\left(\partial_{\gamma} W_{4 d}\right)^{2}+\frac{1}{40}\left(\partial_{\lambda} W_{4 d}\right)^{2}+\frac{1}{3}\left(\partial_{\beta} W_{4 d}\right)^{2}+\left(\partial_{\phi} W_{4 d}\right)^{2}\right]-\frac{3}{8} W_{4 d}^{2} .
$$

It can be readily checked that the function:

$$
W_{4 d}=-6 e^{\frac{8}{3} \gamma-4 \lambda-\frac{\beta}{2}}-4 e^{\frac{8}{3} \gamma+6 \lambda-\frac{\beta}{2}}+Q_{c} e^{\frac{20}{3} \gamma-\frac{\beta}{2}}+2 Q_{f} e^{2 \gamma+2 \lambda+\frac{\phi}{2}-\frac{3 \beta}{2}},
$$

solves (4.15). Moreover, one can verify that $W_{4 d}$ gives rise to the BPS equations satisfied by the zero temperature supersymmetric solution of [25].

In terms of $W_{4 d}$ the boundary action takes the form:

$$
S_{\text {boundary }}=\frac{V_{5} V_{x^{3}}}{2 \kappa_{10}^{2}} \int_{r \rightarrow \infty} d^{3} x \sqrt{\gamma}\left(2 K+W_{4 d}\right),
$$

where $\gamma$ is the determinant of the induced metric on constant- $r$ slices and $K=K_{\mu}^{\mu}$ is the trace of the extrinsic curvature of these slices. One can check that, after diving by the infinite volume $V_{3}$ of the $2+1$ dimensional Minkowski spacetime, the sum of the actions (4.2) and (4.17) evaluated on-shell is finite. We get:

$$
\frac{S_{\text {renormalized }}}{V_{3} V_{x^{3}}}=\frac{S_{\text {eff,on-shell }}+S_{\text {boundary,on-shell }}}{V_{3} V_{x^{3}}}=\frac{3}{7} \beta_{s} Q_{f}^{\frac{2}{3}} Q_{c}^{\frac{5}{3}} T^{\frac{10}{3}}
$$

where $\beta_{s}$ is the constant defined in (3.17). To obtain (4.18) we have integrated from $r=r_{h}$ to $r=\infty$. Notice that $S_{\text {renormalized }}$ is equal, as it should, to minus the free energy density $f$ (compare with (3.20)). The minus sign in this relation is due to the fact that we are working in Minkowski signature.

By taking the functional derivative of the on-shell renormalized action with respect to the boundary metric we obtain the expectation value of the field theory stress-energy tensor:

$$
\left\langle T^{\mu}{ }_{\nu}\right\rangle=\frac{V_{5} V_{x^{3}}}{2 \kappa_{10}^{2}} \sqrt{\gamma}\left[-2 K^{\mu}{ }_{\nu}+\delta^{\mu}{ }_{\nu}\left(2 K+W_{4 d}\right)\right]_{r \rightarrow \infty} .
$$

Evaluating the right-hand side of (4.19) for our solution, we get:

$$
\left\langle T^{\mu}{ }_{\nu}\right\rangle=\operatorname{diag}\left(-\epsilon, p_{x y}, p_{x y}\right),
$$

where $\epsilon$ is the ADM energy density (3.16) and $p_{x y}$ is the pressure in the $x y$ plane written in (3.28). 


\section{$5 \quad$ Effective action in $5 \mathrm{~d}$}

Let us now reduce our system to five dimensions, namely those corresponding to the coordinates $z^{m}=\left(t, x^{1}, x^{2}, x^{3}, r\right)$. In principle this reduction would allow us to study the inter-layer properties and could be used to analyze the consequences of the anisotropy of the model. In this section we will use this $5 \mathrm{~d}$ formalism to compute the complete stress-energy tensor and to establish the holographic dictionary for the D5-brane chemical potential.

Let us adopt the following reduction ansatz for the metric:

$$
d s_{10}^{2}=e^{\frac{10}{3} \gamma} g_{p q} d z^{p} d z^{q}+e^{-2(\gamma+\lambda)} d s_{\mathbb{C P}^{2}}^{2}+e^{2(4 \lambda-\gamma)}(d \tau+A)^{2},
$$

where $g_{p q}$ is a $5 \mathrm{~d}$ metric and the scalar fields $\gamma$ and $\lambda$ depend on the $5 \mathrm{~d}$ coordinates $z^{m}$. It is important to notice that the RR three form $F_{3}$ for our solution has a leg in $x^{3}$, as well as two legs in the internal space (see (A.6)). Therefore, when it is reduced to $5 \mathrm{~d}$ it gives rise to a one-form $\mathcal{F}_{1}$, which we will represent in terms of a scalar potential $\mathcal{V}$ as:

$$
\mathcal{F}_{1}=d \mathcal{V} .
$$

Moreover, our D5-branes are codimension-one objects (extended along the hypersurface $x^{3}=$ constant and smeared over $x^{3}$ ). The corresponding DBI action contains the determinant of the induced metric on this $4 \mathrm{~d}$ surface, which we will denote by $\hat{g}_{4}$, integrated over $x_{3}$ to take into account the smearing. In addition to the metric and $\mathcal{V}$, the $5 \mathrm{~d}$ theory has three scalar fields $(\gamma, \lambda$ and the dilaton $\phi)$. The total effective action is worked out in appendix $\mathrm{C}$ and takes the form:

$$
\begin{aligned}
S_{\text {eff }}= & \frac{V_{5}}{2 \kappa_{10}^{2}} \int d^{5} z \sqrt{-g_{5}}\left[R_{5}-\frac{40}{3}(\partial \gamma)^{2}-20(\partial \lambda)^{2}-\frac{1}{2}(\partial \phi)^{2}-\frac{1}{2} e^{4 \gamma+4 \lambda+\phi}(\partial \mathcal{V})^{2}-U\right] \\
& -\frac{V_{5}}{2 \kappa_{10}^{2}} \int d^{5} z \sqrt{-\hat{g}_{4}}\left[6 Q_{f} e^{\frac{14}{3} \gamma-2 \lambda+\frac{\phi}{2}}\right],
\end{aligned}
$$

where $U$ is the potential:

$$
U=4 e^{\frac{16}{3} \gamma+12 \lambda}-24 e^{\frac{16}{3} \gamma+2 \lambda}+\frac{Q_{c}^{2}}{2} e^{\frac{40}{3} \gamma} .
$$

For our D3-D5 black hole solution the 5d metric takes the form:

$$
d s_{5}^{2}=-d_{1}^{2}(r) d t^{2}+d_{2}^{2}(r)\left[\left(d x^{1}\right)^{2}+\left(d x^{2}\right)^{2}\right]+d_{3}^{2}(r)\left(d x^{3}\right)^{2}+d_{r}^{2}(r)(d r)^{2},
$$

where the different $d$ functions are given by:

$$
\begin{aligned}
& d_{1}^{2}(r)=\left(\frac{9}{8}\right)^{2} r^{2} R^{\frac{4}{3}} b(r) \\
& d_{2}^{2}(r)=\left(\frac{9}{8}\right)^{2} r^{2} R^{\frac{4}{3}} \\
& d_{3}^{2}(r)=\left(\frac{9}{8}\right)^{2}\left(\frac{4 Q_{f}}{3}\right)^{\frac{4}{3}} R^{\frac{4}{3}} r^{\frac{2}{3}}, \\
& d_{r}^{2}(r)=\left(\frac{9}{8}\right)^{2} \frac{R^{\frac{16}{3}}}{r^{2} b(r)} .
\end{aligned}
$$


In (5.6) the function $b(r)$ is the blackening function (2.4). Moreover, the scalar fields corresponding to the D3-D5 black hole are:

$$
e^{\phi}=\left(\frac{3}{4 Q_{f}}\right)^{\frac{2}{3}} r^{\frac{2}{3}}, \quad e^{\gamma}=\left(\frac{8}{9}\right)^{\frac{3}{5}} \frac{1}{R}, \quad e^{\lambda}=\left(\frac{9}{8}\right)^{\frac{1}{10}} .
$$

Notice that they are the same as in (4.11). The function $\mathcal{V}$ is given by:

$$
\mathcal{V}=\sqrt{2} Q_{f} x^{3}
$$

It can be easily checked that the metric written in (5.5) and (5.6), together with the scalars written in (5.7) and the function $\mathcal{V}$ written in (5.8), satisfy the equations of motion derived from the action (5.3) (these equations have been explicitly written in appendix C).

It is also interesting to relate these fields to the ones corresponding to the $4 \mathrm{~d}$ approach for our solution. The $5 \mathrm{~d}$ to $4 \mathrm{~d}$ reduction is analyzed in appendix $\mathrm{C}$ (section C.3). As mentioned above, the scalars $(\phi, \gamma, \lambda)$ take the same values in $4 \mathrm{~d}$ and $5 \mathrm{~d}$. Moreover, the $4 \mathrm{~d}$ scalar $\beta$ is related to $d_{3}$ as:

$$
e^{\beta}=d_{3},
$$

while the functions $c_{1}, c_{2}$ and $c_{3}$ of the $4 \mathrm{~d}$ metric are related to the $d$ functions as:

$$
c_{1}^{2}=d_{3} d_{1}^{2}, \quad c_{2}^{2}=d_{3} d_{2}^{2}, \quad c_{3}^{2}=d_{3} d_{r}^{2}
$$

\subsection{Stress-energy tensor}

Let us now construct boundary counterterms which regularize the on-shell effective action and allow to implement the holographic renormalization formalism and compute the VEV of the stress-energy tensor. First of all we obtain a superpotential $W_{5 d}$ for the potential $U$ written in (5.4). This superpotential must satisfy the equation:

$$
U=\frac{1}{2}\left[\frac{3}{80}\left(\partial_{\gamma} W_{5 d}\right)^{2}+\frac{1}{40}\left(\partial_{\gamma} W_{5 d}\right)^{2}+\left(\partial_{\phi} W_{5 d}\right)^{2}\right]-\frac{1}{3} W_{5 d}^{2}
$$

which is solved by the function:

$$
W_{5 d}=-6 e^{\frac{8 \gamma}{3}-4 \lambda}-4 e^{\frac{8 \gamma}{3}+6 \lambda}+Q_{c} e^{\frac{20}{3} \gamma} .
$$

Notice that the three terms on the right-hand side of (5.12) are in one-to-one correspondence with the terms in the $4 \mathrm{~d}$ superpotential $W_{4 d}$ which do not contain $Q_{f}$ (see (4.16)). Let us next define a new function $W_{\text {flavor }}$, related to the last term in (4.16), as:

$$
W_{\text {flavor }}=2 Q_{f} e^{2 \lambda+2 \gamma+\frac{\phi}{2}} .
$$

The counterterms needed to renormalize the action (5.3) will have the same structure as $S_{\text {eff }}$. First of all, we will have a $5 \mathrm{~d}$ part, containing the metric $\gamma_{a b}$ induced on constant $r$ slices, as well as the Gibbons-Hawking term and the 5d superpotential (5.12). In addition, 
we will have a $4 \mathrm{~d}$ part corresponding to the smeared sources, which contains the determinant of the metric $\hat{\gamma}_{a b}$ induced on constant $r$ and constant $x^{3}$ slices. We construct this term by using the flavor function defined in (5.13). The total boundary action is:

$$
S_{\text {boundary }}=\frac{V_{5}}{2 \kappa_{10}^{2}} \int_{r \rightarrow \infty} d^{4} x \sqrt{\gamma}\left(2 K+W_{5 d}\right)+\frac{V_{5}}{2 \kappa_{10}^{2}} \int_{r \rightarrow \infty} d^{4} x \sqrt{\hat{\gamma}} W_{\text {flavor }} .
$$

One can check that the addition of $S_{\text {boundary }}$ makes the total on-shell action (divided by $\left.V_{3} V_{x^{3}}\right)$ finite. Actually, one has:

$$
\frac{S_{\text {renormalized }}}{V_{3} V_{x^{3}}}=\frac{S_{\text {eff,on-shell }}+S_{\text {boundary,on-shell }}}{V_{3} V_{x^{3}}}=\frac{3}{7} \beta_{s} Q_{f}^{\frac{2}{3}} Q_{c}^{\frac{5}{3}} T^{\frac{10}{3}} .
$$

Notice that $S_{\text {renormalized }} / V_{3} V_{x^{3}}$ coincides with minus the free energy density $f$ in the tendimensional approach (see (3.20)), as it should.

The VEV of the stress-energy tensor of the dual theory can be obtained by taking the functional derivative of $S_{\text {renormalized }}$ with respect to the boundary metric. As a result of this calculation we get contributions from the two types of terms in (5.14):

$$
\left\langle T_{\nu}^{\mu}\right\rangle=\frac{V_{5}}{2 \kappa_{10}^{2}} \sqrt{\gamma}\left[-2 K_{\nu}^{\mu}+\delta_{\nu}^{\mu}\left(2 K+W_{5 d}\right)\right]_{r \rightarrow \infty}+\left\langle T_{\nu}^{\mu}\right\rangle_{\text {flavor }}
$$

where $\left\langle T^{\mu}{ }_{\nu}\right\rangle_{\text {flavor }}$ is only non-vanishing if both indices $\mu$ and $\nu$ take values $0,1,2$ and, in this case, is given by:

$$
\left\langle T^{\mu}{ }_{\nu}\right\rangle_{\text {flavor }}=Q_{f} \frac{V_{5}}{\kappa_{10}^{2}} \sqrt{\hat{\gamma}} e^{2 \lambda+2 \gamma+\frac{\phi}{2}} \delta^{\mu}{ }_{\nu \rightarrow \infty}, \quad \quad \mu, \nu=0,1,2 .
$$

One can easily verify that $\left\langle T^{\mu}{ }_{\nu}\right\rangle$ is given by the same expression as in the 10d analysis, namely by (3.54) with $\epsilon, p_{x y}$ and $p_{z}$ equal to the values written in (3.16) and (3.28).

\subsection{Holographic dictionary}

Clearly, the contribution (5.17) is essential to reproduce the different values of the two pressures $p_{x y}$ and $p_{z}$, i.e., to correctly represent the anisotropic behavior of the model. As argued in section 3, this anisotropy is characterized by the D5-brane chemical potential $\Phi$. It is therefore very important to find a dictionary allowing us to read the value of $\Phi$ from the value of some supergravity field at the UV boundary. This is the purpose of this subsection.

In our holographic setup $\Phi$ should be related to the value of the potential under which the D5-branes are electrically charged. Notice that the D5-branes in our reduced theory extend along $x^{0} x^{1} x^{2}$ and are smeared along $x^{3}$. Therefore, we expect $\Phi$ to be extracted from the components of a three-form $\mathcal{C}_{3}$ along $x^{0} x^{1} x^{2}$. One can find $\mathcal{C}_{3}$ by the following argument. First of all, we write the equation of motion of $\mathcal{F}_{1}$ (eq. (C.25)) as:

$$
d\left(e^{4 \gamma+4 \lambda+\phi} * \mathcal{F}_{1}\right)=0,
$$

where $*$ denotes the Hodge dual of the 5 d theory. Next, we interpret (5.18) as a Bianchi identity, i.e., as the closure of the four-form $\mathcal{F}_{4}$ defined as:

$$
\mathcal{F}_{4}=e^{4 \gamma+4 \lambda+\phi} * \mathcal{F}_{1} .
$$


It follows that $\mathcal{F}_{4}$ can be represented in terms of a three-form $\mathcal{C}_{3}$ :

$$
\mathcal{F}_{4}=d \mathcal{C}_{3},
$$

and we will soon verify that $\mathcal{C}_{3}$ is the three-form we are seeking. To check this statement we will find the form for our solution. First of all we notice that:

$$
* \mathcal{F}_{1}=\sqrt{2} Q_{f} \frac{d_{1} d_{2}^{2} d_{r}}{d_{3}} d x^{0} \wedge d x^{1} \wedge d x^{2} \wedge d r .
$$

From (5.21) we can readily verify that $\mathcal{C}_{3}$ can be taken as:

$$
\mathcal{C}_{3}=\mathcal{A}\left(Q_{f}^{-\frac{1}{3}} r^{\frac{10}{3}}+\mathcal{C}\right) d x^{0} \wedge d x^{1} \wedge d x^{2},
$$

where $\mathcal{A}$ is a known numerical constant (independent of $Q_{f}$ and $Q_{c}$ ) and $\mathcal{C}$ is another constant which we will fix by requiring regularity at the horizon or, equivalently by demanding the vanishing of $\mathcal{C}_{3}$ at $r=r_{h}$. This condition leads to the following value of $\mathcal{C}$ :

$$
\mathcal{C}=-Q_{f}^{-\frac{1}{3}} r_{h}^{\frac{10}{3}}
$$

Taking into account that $r_{h}^{\frac{10}{3}} \propto Q_{c}^{\frac{5}{3}} T^{\frac{10}{3}}$, we find:

$$
\mathcal{C} \propto Q_{c}^{\frac{5}{3}} Q_{f}^{-\frac{1}{3}} T^{\frac{10}{3}}
$$

By comparing (5.24) and (3.24) we conclude that the chemical potential $\Phi$ and the constant $\mathcal{C}$ are proportional:

$$
\Phi \propto \mathcal{C} .
$$

Notice also that $\mathcal{C}$ is (proportional to) the subleading term in the expansion of the $x^{0} x^{1} x^{2}$ component of $\mathcal{C}_{3}$ near the boundary. This identification of $\Phi$ is similar to the one obtained in [27] for the case of an anisotropic background generated by D7-branes. The fact that is the subleading term that is being identified with $\Phi$, and not the leading term as in other holographic setups, can be traced back to the Hodge duality that we are doing when passing from $\mathcal{F}_{1}$ to $\mathcal{F}_{4}$.

\section{$6 \quad$ Fluctuations and hydrodynamics}

We will now explore the hydrodynamic properties of our system. In particular we will compute the transport coefficients for perturbations propagating along the $x^{1} x^{2}$ plane. The purpose of this calculation is to characterize the effects of flavors, and of the corresponding induced anisotropy, on the transport properties of our system. As already mentioned in the introduction, our main result is that the transport coefficients in the $x^{1} x^{2}$ plane are the same as those of a D2-brane. This result confirms the conclusions of our static thermodynamic analysis and implies that, in our model, the dynamics of the excitations within a layer is governed by an effective strongly coupled super Yang-Mills theory in $2+1$ dimensions. 
Following the standard procedure [47], we have to study the fluctuations of the $4 \mathrm{~d}$ metric and scalar fields around their background values (4.10) and (4.11). In order to do this, we will perform the following substitution in the equations of motion:

$$
g_{m n} \rightarrow g_{m n}+h_{m n}, \quad \Psi \rightarrow \Psi+\delta \Psi,
$$

for $\Psi=(\phi, \gamma, \lambda, \beta)$ and we will keep only the first-order terms in $h_{m n}$ and $\delta \Psi$. Moreover, we will work in the radial gauge for the metric, in which:

$$
h_{m r}=0, \quad\left(m=t, x^{1}, x^{2}, r\right) .
$$

Let us start by computing the variation of the scalar equation (4.8). One can easily check that, at first order, we have:

$$
\delta \square \Psi=\square \delta \Psi+\frac{1}{2} g^{m n} \partial_{m} \Psi \partial_{n}\left(h_{p}^{p}\right)-\frac{1}{\sqrt{-g_{4}}} \partial_{m}\left(\sqrt{-g_{4}} h^{m n} \partial_{n} \Psi\right) .
$$

The last term in (6.3) is always zero in the radial gauge when the scalar fields of the background only depend on the radial variable. For a metric of the type (4.9), $\square \delta \Psi$ becomes:

$$
\square \delta \Psi=\frac{1}{c_{3}^{2}}\left[\partial_{r}^{2}(\delta \Psi)+\partial_{r} \log \left(\frac{c_{1} c_{2}^{2}}{c_{3}}\right) \partial_{r}(\delta \Psi)\right]-\frac{\partial_{t}^{2}(\delta \Psi)}{c_{1}^{2}}+\frac{\partial_{x^{1}}^{2}(\delta \Psi)+\partial_{x^{2}}^{2}(\delta \Psi)}{c_{2}^{2}},
$$

and the first-order equation for $\delta \Psi$ is:

$$
\begin{aligned}
\partial_{r}^{2}(\delta \Psi)+\partial_{r} \log \left(\frac{c_{1} c_{2}^{2}}{c_{3}}\right) \partial_{r}(\delta \Psi)-\frac{c_{3}^{2}}{c_{1}^{2}} \partial_{t}^{2}(\delta \Psi)+\frac{c_{3}^{2}}{c_{2}^{2}}\left(\partial_{x^{1}}^{2}(\delta \Psi)+\partial_{x^{2}}^{2}(\delta \Psi)\right) \\
+\frac{\partial_{r} \Psi}{2} \partial_{r}\left(\frac{h_{x^{1} x^{1}}+h_{x^{2} x^{2}}}{c_{2}^{2}}-\frac{h_{t t}}{c_{1}^{2}}\right)=c_{3}^{2} \alpha_{\Psi} \delta\left[\partial_{\Psi} V\right] .
\end{aligned}
$$

The first-order variation of the Einstein equation (4.6) is:

$$
\delta R_{m n}=\sum_{\Psi} \frac{1}{2 \alpha_{\Psi}}\left(\partial_{m}(\delta \Psi) \partial_{n} \Psi+\partial_{m} \Psi \partial_{n}(\delta \Psi)\right)+\frac{1}{2} h_{m n} V+\frac{1}{2} g_{m n} \delta V
$$

where $\delta R_{m n}$ can be written in terms of covariant derivatives of the metric perturbation $h_{m n}$ as:

$$
\delta R_{m n}=\frac{1}{2}\left[D_{p} D_{m} h_{n}^{p}+D_{p} D_{n} h_{m}^{p}-D_{p} D^{p} h_{m n}-D_{m} D_{n} h_{p}^{p}\right]
$$

By plugging (6.7) into (6.6), we arrive at the following equation for the metric fluctuations:

$$
\begin{aligned}
& D_{p} D_{m} h_{n}^{p}+D_{p} D_{n} h_{m}^{p}-D_{p} D^{p} h_{m n}-D_{m} D_{n} h_{p}^{p}= \\
& \quad=\sum_{\Psi} \frac{1}{\alpha_{\Psi}}\left(\partial_{m}(\delta \Psi) \partial_{n} \Psi+\partial_{m} \Psi \partial_{n}(\delta \Psi)\right)+h_{m n} V+g_{m n} \delta V
\end{aligned}
$$




\subsection{The shear channel}

The fluctuation equations (6.5) and (6.8) are highly coupled. However, one can identify several consistent truncations in which only few fluctuations are non-zero. Without loss of generality, let us consider a perturbation propagating along the $x^{2}$ direction. The first of the consistent truncations that we will analyze is the so-called shear channel, in which only the metric fluctuations $h_{t x^{1}}$ and $h_{x^{1} x^{2}}$ are excited. Let us assume that these fluctuations have frequency $\omega$ and momentum $q$ and, accordingly, let us parametrize them as:

$$
\begin{aligned}
h_{t x^{1}} & =e^{-i\left(\omega t-q x^{2}\right)} c_{2}^{2}(r) H_{t x}(r), \\
h_{x^{1} x^{2}} & =e^{-i\left(\omega t-q x^{2}\right)} c_{2}^{2}(r) H_{x y}(r),
\end{aligned}
$$

where $c_{2}(r)$ is the function written in (4.11) and has been included in the ansatz (6.9) for convenience. The equations of motion of $H_{t x}$ and $H_{x y}$ are studied in detail in appendix D. It turns out that they can be reduced to a single second-order differential equation for a gauge invariant combination $X$, defined as:

$$
X \equiv q H_{t x}+\omega H_{x y}
$$

The equation satisfied by $X$ is:

$$
X^{\prime \prime}+\frac{(10+3 b(r)) \omega^{2}-13 b^{2}(r) q^{2}}{3 b(r) r\left(\omega^{2}-b(r) q^{2}\right)} X^{\prime}+\frac{R^{4}}{r^{4} b^{2}(r)}\left(\omega^{2}-b(r) q^{2}\right) X=0 .
$$

Let us now work in a new radial variable $x$, related to $r$ as:

$$
x=[b(r)]^{\frac{1}{2}} .
$$

In this new variable the horizon is located at $x=0$, whereas the boundary is at $x=1$. We will consider the gauge-invariant combination $X$ as a function of $x$. Moreover, it is quite convenient to introduce the dimensionless momentum and frequency $\hat{q}$ and $\hat{\omega}$, defined as:

$$
\hat{q}=\frac{q}{2 \pi T}, \quad \hat{\omega}=\frac{\omega}{2 \pi T} .
$$

Then, if the prime now denotes derivatives with respect to $x$, eq. (6.11) takes the form:

$$
X^{\prime \prime}-\frac{1}{x} \frac{\hat{q}^{2} x^{2}+\hat{\omega}^{2}}{\hat{q}^{2} x^{2}-\hat{\omega}^{2}} X^{\prime}-\frac{\hat{q}^{2} x^{2}-\hat{\omega}^{2}}{x^{2}\left(1-x^{2}\right)^{\frac{7}{5}}} X=0 .
$$

We want to solve (6.14) by imposing infalling boundary conditions at the horizon $x=0$, as well as Dirichlet boundary conditions at the boundary $x=1$. These solutions only exist when the frequency $\omega$ and the momentum $q$ are related in a particular way, which determines the dispersion relation $\omega=\omega(q)$ of our modes. In the hydrodynamic regime the momentum $q$ is small and one can expand $\omega$ in a power series in $q$. In the shear channel we are studying this relation takes the form:

$$
\omega=-i D_{\eta} q^{2}\left(1+\tau_{s} D_{\eta} q^{2}\right)
$$


where we are keeping terms up to quartic power of $q$. The dispersion relation (6.15) depends on two transport coefficients $D_{\eta}$ and $\tau_{s}$, which we will calculate for our system in this section. We will work in the dimensionless variables defined in (6.13). Moreover, we define the rescaled coefficients $\hat{D}_{\eta}$ and $\hat{\tau}_{s}$ as:

$$
\hat{D}_{\eta}=2 \pi T D_{\eta}, \quad \hat{\tau}_{s}=2 \pi T \tau_{s} .
$$

In terms of the rescaled quantities, the dispersion relation (6.15) takes the form:

$$
\hat{\omega}=-i \hat{D}_{\eta} \hat{q}^{2}\left(1+\hat{\tau}_{s} \hat{D}_{\eta} \hat{q}^{2}\right)
$$

The coefficient $\hat{D}_{\eta}$ determines the ratio of the shear viscosity $\eta$ to the entropy density $s$, namely:

$$
\frac{\eta}{s}=\frac{\hat{D}_{\eta}}{2 \pi}
$$

Below we will find that, for our system, $\hat{D}_{\eta}=1 / 2$, which is equivalent to having $\eta / s=$ $1 /(4 \pi)$. In what follows we compute $\tau_{s}$ explicitly for our system and it turns out that $\tau_{s}$ is the same as the one found in [48] for the geometry of the D2-brane.

Let us come back to the integration of the differential equation (6.14). In order to impose infalling boundary conditions at the horizon $x=0$, we will adopt the ansatz:

$$
X(x)=x^{-i \hat{\omega}} S(x),
$$

where $S(x)$ must be regular at $x=0$. Let us expand $S(x)$ in powers of $\hat{q}$ as:

$$
S(x)=S_{0}(x)+\hat{q}^{2} S_{2}(x)+\cdots .
$$

Plugging the expansions (6.20) and (6.15) into (6.14) and separating the different orders in $\hat{q}$, we get the following system of equations:

$$
\begin{aligned}
& S_{0}^{\prime \prime}-\frac{1}{x} S_{0}^{\prime}=0 \\
& S_{2}^{\prime \prime}-\frac{1}{x} S_{2}^{\prime}=\left(\frac{1}{\left(1-x^{2}\right)^{\frac{7}{5}}}-\frac{2 \hat{D}_{\eta}}{x^{2}}\right) S_{0}+\frac{2 \hat{D}_{\eta}}{x}\left(1-\frac{\hat{D}_{\eta}}{x^{2}}\right) S_{0}^{\prime} .
\end{aligned}
$$

We can also expand $S(x)$ in powers of $x$ near $x=0$ :

$$
S(x)=1+\sigma_{2} x^{2}+\sigma_{4} x^{4}+\cdots,
$$

where the coefficients $\sigma_{2}$ and $\sigma_{4}$ are easy to obtain by substituting this expansion into (6.14). They are given by:

$$
\begin{aligned}
\sigma_{2} & =\frac{5 i \hat{q}^{2}(2 i+\hat{\omega})-7 i \hat{\omega}^{3}}{20 \hat{\omega}(i+\hat{\omega})}, \\
\sigma_{4} & =\frac{-25 \hat{q}^{4}(4 i+\hat{\omega})+70 \hat{q}^{2} \hat{\omega}(2 i+\hat{\omega})^{2}+7 \hat{\omega}^{3}\left(24-24 i \hat{\omega}-7 \hat{\omega}^{2}\right)}{800 \hat{\omega}(i+\hat{\omega})(2 i+\hat{\omega})} .
\end{aligned}
$$


By expanding $\sigma_{2}$ and $\sigma_{4}$ in powers of $\hat{q}$ using the dispersion relation (6.15), we arrive at the following expression of $S(x)$, valid for low $x$ and low $\hat{q}$ :

$$
S(x)=1-\frac{x^{2}}{2 \hat{D}_{\eta}}+\frac{\hat{q}^{2} x^{2}}{80 \hat{D}_{\eta}}\left(20 \hat{D}_{\eta}\left(2 \hat{\tau}_{s}-1\right)+\left(14 \hat{D}_{\eta}-5\right) x^{2}\right)+\mathcal{O}\left(\hat{q}^{3}\right) .
$$

We will next compare (6.24) with the result of integrating the system (6.21) and expanding the result of this integration in powers of $x$ near $x=0$. The integration of the first equation in (6.21) is straightforward and yields the result:

$$
S_{0}(x)=A+B x^{2},
$$

where $A$ and $B$ are integration constants. By comparing (6.25) with the first two terms in (6.24) we conclude that $A=1$ and $B=-1 /\left(2 \hat{D}_{\eta}\right)$ and, therefore, $S_{0}(x)$ is given by:

$$
S_{0}(x)=1-\frac{x^{2}}{2 \hat{D}_{\eta}} .
$$

By imposing the Dirichlet condition $S_{0}(x=1)=0$ at the boundary, we obtain that, as already announced, $\hat{D}_{\eta}$ must be:

$$
\hat{D}_{\eta}=\frac{1}{2}
$$

and $S_{0}$ takes the form:

$$
S_{0}(x)=1-x^{2} .
$$

Using these values of $S_{0}(x)$ and $\hat{D}_{\eta}$ on the right-hand side of the second equation of the system (6.21) we arrive at the equation:

$$
S_{2}^{\prime \prime}-\frac{1}{x} S_{2}^{\prime}=\frac{1}{\left(1-x^{2}\right)^{\frac{2}{5}}}-1
$$

whose general solution is:

$$
S_{2}(x)=C+(1+2 D-2 \log x) \frac{x^{2}}{4}-\frac{25}{24} e^{\frac{2 \pi i}{5}} x^{\frac{6}{5}} F\left(-\frac{3}{5}, \frac{2}{5} ; \frac{7}{5} ; \frac{1}{x^{2}}\right) .
$$

In (6.30) $C$ and $D$ are integration constants which can be determined by expanding the result near $x \approx 0$ and comparing it with the terms proportional to $\hat{q}^{2}$ of (6.24). The expansion of $(6.30)$ near $x \approx 0$ is:

$$
S_{2}(x)=C+\frac{5}{12}+\frac{x^{2}}{2}\left[D+\frac{1}{2}\left(\gamma-i \pi+\psi\left(\frac{2}{5}\right)\right)\right]+\frac{x^{4}}{20}+\cdots,
$$

where $\gamma \approx .577$ is the Euler-Mascheroni constant and $\psi(z)$ is the logarithmic derivative of the Euler gamma function $\Gamma(z)$. This result coincides with (6.24) if the constants $C$ and $D$ are:

$$
\begin{aligned}
& C=-\frac{5}{12} \\
& D=-\frac{1}{2}\left[1-2 \hat{\tau}_{s}+\gamma-i \pi+\psi\left(\frac{2}{5}\right)\right] .
\end{aligned}
$$


Substituting (6.32) in (6.30) we get the function $S_{2}(x)$, namely:

$$
S_{2}(x)=-\frac{5}{12}+\left(2 \hat{\tau}_{s}-\gamma+i \pi-\psi\left(\frac{2}{5}\right)-2 \log x\right) \frac{x^{2}}{4}-\frac{25}{24} e^{\frac{2 \pi i}{5}} x^{\frac{6}{5}} F\left(-\frac{3}{5}, \frac{2}{5} ; \frac{7}{5} ; \frac{1}{x^{2}}\right),
$$

which only contains $\hat{\tau}_{s}$ as unknown parameter. By imposing that $S_{2}(x=1)=0, \hat{\tau}_{s}$ is fixed to be:

$$
\hat{\tau}_{s}=\frac{1}{2}\left[\gamma+\psi\left(\frac{8}{5}\right)\right]
$$

Equivalently, the unrescaled parameter is:

$$
\tau_{s}=\frac{1}{4 \pi T}\left[\gamma+\psi\left(\frac{8}{5}\right)\right] .
$$

This value of $\tau_{s}$ coincides with the one found in the literature for the D2-brane [48].

\subsection{The sound channel}

In the so-called sound channel, the following set of metric fluctuations, propagating along $x^{2}$, are decoupled from the others:

$$
\left(h_{t t}, h_{t x^{2}}, h_{x^{1} x^{1}}, h_{x^{2} x^{2}}\right)
$$

and are coupled to the fluctuations of the scalar fields. Let us parametrize these metric fluctuations as:

$$
\begin{aligned}
h_{t t} & =e^{-i\left(\omega t-q x^{2}\right)} c_{1}^{2}(r) H_{t t}(r), & h_{t x^{2}} & =e^{-i\left(\omega t-q x^{2}\right)} c_{2}^{2}(r) H_{t y}(r), \\
h_{x^{1} x^{1}} & =e^{-i\left(\omega t-q x^{2}\right)} c_{2}^{2}(r) H_{x x}(r), & h_{x^{2} x^{2}} & =e^{-i\left(\omega t-q x^{2}\right)} c_{2}^{2}(r) H_{y y}(r),
\end{aligned}
$$

where $c_{1}(r)$ and $c_{2}(r)$ are the functions written in (4.10). Similarly, we represent the scalar fluctuations as:

$$
\begin{array}{llrl}
\delta \phi & =e^{-i\left(\omega t-q x^{2}\right)} \Phi(r), & & \delta \gamma=e^{-i\left(\omega t-q x^{2}\right)} \Gamma(r), \\
\delta \lambda=e^{-i\left(\omega t-q x^{2}\right)} \Lambda(r), & \delta \beta & =e^{-i\left(\omega t-q x^{2}\right)} B(r) .
\end{array}
$$

Let us now introduce a compact notation for the scalar fluctuations. We denote by $\hat{\Psi}(r)$ the radial part of the fluctuation $\Psi=(\phi, \gamma, \lambda, \beta)$, namely:

$$
\hat{\Psi}(r)=(\Phi(r), \Gamma(r), \Lambda(r), B(r)) .
$$

Then, (6.38) can be rewritten simply as:

$$
\delta \Psi=e^{-i\left(\omega t-q x^{2}\right)} \hat{\Psi}(r) .
$$

The full set of equations for the fields of (6.37) and (6.38) is written in appendix D. As usual, these equations are highly redundant due to the diffeomorphism gauge invariance. This redundancy can be reduced by defining new fields. Accordingly, let us define new 
scalar fluctuation fields $Z_{\Phi}, Z_{\Gamma}, Z_{\Lambda}$ and $Z_{B}$, denoted collectively by $Z_{\hat{\Psi}}$, as the following combination of $\hat{\Psi}$ and $H_{x x}$ :

$$
Z_{\hat{\Psi}}=\hat{\Psi}-\frac{\Psi^{\prime}}{\partial_{r} \log c_{2}^{2}} H_{x x}
$$

As argued in $[44,49,50]$, these are the gauge invariant combinations of the scalar fields and the metric. It is proved in appendix $\mathrm{D}$ that the equations for the $Z$ 's close among themselves (see the system (D.12)). Moreover, there is a particular combination $Z_{S}$ of these fields which can be decoupled from the other scalars. This combination is:

$$
Z_{S}(r) \equiv 3 Z_{B}(r)+2 Z_{\Phi}(r) .
$$

The equation satisfied by $Z_{S}(r)$ has been written in (D.14). Following [47], we now define the gauge invariant metric fluctuation $Z_{H}$ as:

$$
Z_{H}=H_{y y}+\frac{2 q}{\omega} H_{t y}+\frac{q^{2}}{\omega^{2}} \frac{c_{1}^{2}}{c_{2}^{2}} H_{t t}+\left(\frac{q^{2}}{\omega^{2}} \frac{c_{1}^{2} \partial_{r} \log c_{1}}{c_{2}^{2} \partial_{r} \log c_{2}}-1\right) H_{x x} .
$$

The equation satisfied by $Z_{H}$ has been written in (D.15). This equation shows that $Z_{H}$ is only coupled to $Z_{S}$. Since $Z_{S}$ does not couple to any other scalar, we can start our analysis by finding $Z_{S}$ and then using this result in the equation for $Z_{H}$. It is shown in appendix D that the only acceptable solution for $Z_{S}$ is the trivial one $Z_{S}=0$. Thus, we are left with a single equation for the gauge invariant metric fluctuation $Z_{H}$. Let us adopt for $Z_{H}$ an ansatz similar to the one used for the fluctuations in the shear channel, namely:

$$
Z_{H}(r)=[b(r)]^{-\frac{i \hat{\omega}}{2}} Y(r) .
$$

Furthermore, we will work in the $x$ variable defined in (6.12). After some work one can verify that the equation satisfied by $Y(x)$ is:

$$
\begin{gathered}
Y^{\prime \prime}+\frac{\left[5-2(3+2 i \hat{\omega}) x^{2}-10 i \hat{\omega}\right] \hat{q}^{2}+7(2 i \hat{\omega}-1) \hat{\omega}^{2}}{x\left[\left(5+2 x^{2}\right) \hat{q}^{2}-7 \hat{\omega}^{2}\right]} Y^{\prime}+ \\
+\left[-\frac{\hat{q}^{2}}{\left(1-x^{2}\right)^{\frac{7}{5}}}+\frac{1}{x^{2}}\left(\frac{1}{\left(1-x^{2}\right)^{\frac{7}{5}}}-1\right) \hat{\omega}^{2}+\frac{8(1+i \hat{\omega}) \hat{q}^{2}}{\left(5+2 x^{2}\right) \hat{q}^{2}-7 \hat{\omega}^{2}}\right] Y=0,
\end{gathered}
$$

where the primes denote derivative with respect to the new variable $x$.

We want to integrate the differential equations for $Y(x)$ in the hydrodynamic limit of low momentum. We will impose infalling boundary conditions at the horizon for $Z_{H}(r)$ and we will demand that the fluctuations vanish at the boundary. The infalling boundary condition at the horizon $x=0$ is equivalent to the regularity of $Y(x)$ at this point. These conditions would require a specific dispersion relation $\omega=\omega(q)$, which at low momentum can be expanded as:

$$
\omega=v_{s} q-i \Gamma q^{2}+\mathcal{T} q^{3}
$$

where we have only kept terms up to third order in $q$. The coefficient $v_{s}$ of the linear term in (6.46) is the speed of sound and the quadratic coefficient $\Gamma$ is the attenuation which, in $p$ spatial dimensions, is related to the shear viscosity $\eta$ and the bulk viscosity $\zeta$ as:

$$
\Gamma=\frac{1}{T s}\left[\frac{p-1}{p} \eta+\frac{\zeta}{2}\right]
$$


where $s$ is the entropy density. In our $p=2$ case this expression becomes:

$$
\Gamma=\frac{1}{2 T s}(\eta+\zeta) .
$$

The cubic coefficient $\mathcal{T}$ is usually [51] parametrized as:

$$
\mathcal{T}=\frac{\Gamma}{v_{s}}\left[v_{s}^{2} \tau_{\text {eff }}-\frac{\Gamma}{2}\right],
$$

where $\tau_{\text {eff }}$ is an effective equilibration time which, in $p$ spatial dimensions, is related to the second-order transport coefficients $\tau_{\pi}$ and $\tau_{\Pi}$ of the Israel-Stewart theory as:

$$
\tau_{\text {eff }}=\frac{\tau_{\pi}+\frac{p}{2(p-1)} \frac{\zeta}{\eta} \tau_{\Pi}}{1+\frac{p}{2(p-1)} \frac{\zeta}{\eta}} .
$$

In our $p=2$ model we have:

$$
\tau_{\text {eff }}=\frac{\tau_{\pi}+\frac{\zeta}{\eta} \tau_{\Pi}}{1+\frac{\zeta}{\eta}} .
$$

In what follows it is quite convenient to work with the dimensionless momentum and frequency $\hat{q}$ and $\hat{\omega}$ defined in (6.13). In terms of these rescaled quantities, the dispersion relation (6.46) takes the form:

$$
\hat{\omega}=v_{s} \hat{q}-i \hat{\Gamma} \hat{q}^{2}+\hat{\mathcal{T}} \hat{q}^{3},
$$

where $\hat{\Gamma}$ and $\hat{\mathcal{T}}$ are related to $\Gamma$ and $\mathcal{T}$ as:

$$
\hat{\Gamma}=2 \pi T \Gamma, \quad \hat{\mathcal{T}}=(2 \pi T)^{2} \mathcal{T} .
$$

Let us now analyze (6.45) in the hydrodynamic approximation. We first expand $Y(x)$ in powers of $\hat{q}$ (up to second order) as:

$$
Y(x)=Y_{0}(x)+i \hat{q} Y_{1}(x)+\hat{q}^{2} Y_{2}(x) .
$$

By using (6.54) and (6.46) in (6.45), one can readily show that $Y_{0}(x)$ satisfies the equation:

$$
Y_{0}^{\prime \prime}+\frac{5-7 v_{s}^{2}-6 x^{2}}{x\left(5-7 v_{s}^{2}+2 x^{2}\right)} Y_{0}^{\prime}+\frac{8}{5-7 v_{s}^{2}+2 x^{2}} Y_{0}=0
$$

whose general solution is:

$$
Y_{0}(x)=C_{1}\left(1+\frac{2 x^{2}}{7 v_{s}^{2}-5}\right)+C_{2}\left(2\left(7 v_{s}^{2}-5\right)+\left(2 x^{2}-5+7 v_{s}^{2}\right) \log x\right),
$$

where $C_{1}$ and $C_{2}$ are integration constants. Regularity at the horizon $(x=0)$ requires that $C_{2}=0$. By imposing that

$$
Y_{0}(x=1)=0,
$$

we get the speed of sound $v_{s}$, namely:

$$
v_{s}=\sqrt{\frac{3}{7}}
$$


which coincides with the value we found in our static analysis for the propagation in the $x^{1} x^{2}$ plane, i.e., it is the same as the speed of sound propagating along the gauge theory directions of a D2-brane.

Without loss of generality we can take $C_{1}=1$ (or, equivalently, $Y_{0}(x=0)=1$ ) and, therefore, $Y_{0}(x)$ becomes:

$$
Y_{0}(x)=1-x^{2}
$$

The equation for $Y_{1}$ is:

$$
\begin{aligned}
Y_{1}^{\prime \prime} & +\frac{5-7 v_{s}^{2}-6 x^{2}}{x\left(5-7 v_{s}^{2}+2 x^{2}\right)} Y_{1}^{\prime}+\frac{8}{5-7 v_{s}^{2}+2 x^{2}} Y_{1}= \\
& =\frac{8 v_{s}}{5-7 v_{s}^{2}+2 x^{2}}\left[\frac{14 \hat{\Gamma}}{5-7 v_{s}^{2}+2 x^{2}}-1\right] Y_{0}+\frac{v_{s}}{x}\left[2-\frac{112 x^{2} \hat{\Gamma}}{\left(5-7 v_{s}^{2}+2 x^{2}\right)^{2}}\right] Y_{0}^{\prime} .
\end{aligned}
$$

Using the values of $Y_{0}$ and $v_{s}$ written in (6.59) and (6.58) this equation becomes:

$$
Y_{1}^{\prime \prime}+\frac{1-3 x^{2}}{x\left(1+x^{2}\right)} Y_{1}^{\prime}+\frac{4}{1+x^{2}} Y_{1}=4 \sqrt{\frac{3}{7}} \frac{7 \hat{\Gamma}-2}{1+x^{2}} .
$$

The general solution of $(6.61)$ is:

$$
Y_{1}(x)=\sqrt{\frac{3}{7}}(7 \hat{\Gamma}-2)+C_{1}\left(1-x^{2}\right)+C_{2}\left(4+\left(1-x^{2}\right) \log x^{2}\right),
$$

where, again, $C_{1}$ and $C_{2}$ are integration constants. The regularity requirement at the horizon $x=0$ implies that $C_{2}=0$. Moreover, the UV condition $Y_{1}(x=1)=0$ fixes the rescaled attenuation to be:

$$
\hat{\Gamma}=\frac{2}{7}
$$

which, according to (6.53), is equivalent to the following value of $\Gamma$ :

$$
\Gamma=\frac{1}{7 \pi T}
$$

Taking into account that $\eta / s=1 / 4 \pi$, it follows from (6.64) and (6.48) that the ratio of the bulk and shear viscosities for our model is:

$$
\frac{\zeta}{\eta}=\frac{1}{7}
$$

This value for $\zeta / \eta$ is exactly the same as the one corresponding to a D2-brane [44], which saturates Buchel's bound [52]:

$$
\frac{\zeta}{\eta}=2\left(\frac{1}{2}-v_{s}^{2}\right)
$$

Let us next look at the equation for $Y_{2}(x)$. Using the values of $v_{s}$ and $\hat{\Gamma}$ already determined, this equation reduces to:

$$
Y_{2}^{\prime \prime}+\frac{1-3 x^{2}}{x\left(1+x^{2}\right)} Y_{2}^{\prime}+\frac{4}{1+x^{2}} Y_{2}=g(x),
$$


where $g(x)$ is the following function:

$$
g(x)=\frac{1}{\left(1-x^{2}\right)^{\frac{2}{5}}}-\frac{3}{7}\left(1-\frac{1}{x^{2}}+\frac{1}{x^{2}\left(1-x^{2}\right)^{\frac{2}{5}}}\right)-\frac{4}{7} \frac{7 \sqrt{21} \hat{\mathcal{T}}-4}{1+x^{2}} .
$$

The homogeneous equation in (6.67) is just the same as in (6.61). We already found two independent solutions in (6.62), which we now denote by $y_{1}(x)$ and $y_{2}(x)$ :

$$
y_{1}(x)=1-x^{2}, \quad y_{2}(x)=\left(1-x^{2}\right) \log x^{2}+4 .
$$

Then, the general solution of (6.67) can be written as:

$$
Y_{2}(x)=D_{1} y_{1}(x)+D_{2} y_{2}(x)+y_{p}(x)
$$

where $D_{1}$ and $D_{2}$ are constants and $y_{p}(x)$ is a particular solution of the full inhomogeneous equation. We will use the method of variation of constants to find $y_{p}(x)$. The result can be written as:

$$
y_{p}(x)=y_{2}(x) \int d x \frac{y_{1}(x) g(x)}{W(x)}-y_{1}(x) \int d x \frac{y_{2}(x) g(x)}{W(x)},
$$

where $W(x)$ is the Wronskian:

$$
W(x)=y_{1}(x) y_{2}^{\prime}(x)-y_{1}^{\prime}(x) y_{2}(x) .
$$

Let us rewrite (6.71) in a more convenient way following [53, 54]. First of all, we define $h(x)$ as the ratio between the two solutions of the homogeneous equation:

$$
h(x)=\frac{y_{2}(x)}{y_{1}(x)} .
$$

The Wronskian $W(x)$ is related to the derivative of $h(x)$ as:

$$
W(x)=h^{\prime}(x) y_{1}^{2}(x),
$$

and, therefore, we can rewrite (6.71) as:

$$
y_{p}(x)=y_{1}(x)\left[h(x) \int d x \frac{g(x)}{y_{1}(x) h^{\prime}(x)}-\int d x \frac{h(x) g(x)}{h^{\prime}(x) y_{1}(x)}\right] .
$$

After an integration by parts, this equation can be recast as:

$$
y_{p}(x)=y_{1}(x) \int d x h^{\prime}(x) \int^{x} \frac{g(z)}{y_{1}(z) h^{\prime}(z)} d z .
$$

We now impose the regularity condition at the horizon $x=0$. Using the integral expression (6.76) one can show that near $x \rightarrow 0$ the solution behaves as:

$$
Y_{2}(x) \approx \mathcal{A}+\mathcal{B} \log x+\cdots
$$


where $\mathcal{A}$ and $\mathcal{B}$ are constants and the dots represent terms that vanish at $x=0$. Our regularity condition demands that the term with the logarithm be absent in (6.77). Then, we require:

$$
\mathcal{B}=0 \text {. }
$$

This determines the constant $D_{2}$ in (6.70) to be:

$$
D_{2}=\frac{1}{14}\left[\frac{3}{2}\left(\gamma-i \pi+\psi\left(\frac{2}{5}\right)\right)-1\right]
$$

where $\gamma=0.577$ is the Euler-Mascheroni constant and $\psi(x)=\Gamma^{\prime}(x) / \Gamma(x)$ is the digamma function. We next impose the UV boundary condition at $x=1$ :

$$
Y_{2}(x \rightarrow 1)=0
$$

which determines the value of $\hat{\mathcal{T}}$ as:

$$
\hat{\mathcal{T}}=\frac{1}{7} \sqrt{\frac{3}{7}}\left(1+\gamma+\psi\left(\frac{8}{5}\right)\right) .
$$

Numerically, $\hat{\mathcal{T}} \approx 0.1592$. Using (6.53) we find the following value of $\mathcal{T}$ :

$$
\mathcal{T}=\frac{\sqrt{3}}{28 \sqrt{7}(\pi T)^{2}}\left(1+\gamma+\psi\left(\frac{8}{5}\right)\right)
$$

Taking into account the value of $\Gamma$ we found (eq. (6.64)), this result corresponds to having an equilibration time $\tau_{\text {eff }}$ equal to:

$$
\tau_{\mathrm{eff}}=\frac{1}{4 \pi T}\left[\frac{5}{3}+\gamma+\psi\left(\frac{8}{5}\right)\right]
$$

which again coincides with the one found for the geometry of a D2-brane [53, 54]. From this value of $\tau_{\text {eff }}$ we get the following relation between the two Israel-Stewart coefficients, namely:

$$
7 \tau_{\pi}+\tau_{\Pi}=\frac{2}{\pi T}\left[\frac{5}{3}+\gamma+\psi\left(\frac{8}{5}\right)\right]
$$

\section{Summary and conclusions}

Let us summarize our main results. We have succeeded in generalizing the D3-D5 geometry of [25] to include an event horizon. Our solution is analytic and simple and is the gravity dual of the defect theory introduced in [6] at non-zero temperature in the approximation in which the massless flavors are smeared. The geometry found is homogeneous but anisotropic in the gauge theory directions: it preserves translational invariance but breaks rotational symmetry.

We have studied the thermodynamics and hydrodynamics of the model. We have checked several thermodynamic relations and found that the results are consistent with the laws of anisotropic thermodynamics. We also obtained dimensionally reduced gravitational 
actions for our system in four and five dimensions. In both dimensionalities we managed to construct boundary terms to renormalize the on-shell action and find the stress-energy tensor. Moreover, we obtained the hydrodynamic transport coefficients (up to second order) for perturbations propagating in the $x^{1} x^{2}$ plane. These transport coefficients are exactly the same as those of the D2-brane, a result which is not obvious despite the $2+1$ dimensionality of our defect theory.

It follows from our results that the energy of our system scales with $Q_{c}$ and $Q_{f}$ as $Q_{c}^{\frac{5}{3}} Q_{f}^{\frac{2}{3}}$, which determines the dependence of the effective number of degrees of freedom on the number of colors and flavors. This type of dependence with $Q_{c}$ and $Q_{f}$ shows up in our thermodynamic results of section 3 , as well as in the dependence of the entanglement entropy $S_{\|}$(see eq. (2.17)). The non-integer powers of $Q_{c}$ and $Q_{f}$ in this scaling are reflecting the strong coupling regime of the dynamics of the layers. The main result of our thermodynamic and hydrodynamic analysis is that this layer behavior can be reproduced by an effective D2-brane or, equivalently, by $2+1$ super Yang-Mills in the strong coupling regime.

Let us discuss some possible extensions of our work. We could use our entanglement entropy results for slabs of appendix B to study the quantum correlations of the model. From the dependence of the entanglement entropy on the width of the slab it should be immediate to study the mutual information of two slabs and to analyze the possible phase transitions. Moreover, we could also test our geometry with different probe branes, which would correspond to adding new degrees of freedom. One possibility would be adding D5brane probes of the same type as the ones that originated the background and studying their thermodynamics as in [55]. In this probe brane setup it is rather easy to add a baryonic chemical potential. Another possibility would be adding D7-branes extended along the four Minkowski directions, which would allow us to study the anisotropy of the model from a different point of view.

We have restricted our hydrodynamic study to modes propagating in the $x^{1} x^{2}$ plane. It would be very interesting to extend this analysis to modes propagating along $x^{3}$ and to explore the effects of anisotropy on the transport coefficients. To carry out this task we should make use of the $5 \mathrm{~d}$ reduced action found in section 5 . However, this reduced model contains a codimension one object embedded in the fixed hypersurface $x^{3}=$ constant (and smeared over $x^{3}$ ). The fluctuations of this action involving the $x^{3}$ direction are very difficult to treat and we could not find the analogue of the decoupled gauge invariant combinations of section 6. On general grounds we would expect to find the same speed of sound $v_{z}$ as in (3.30). In the shear channel we could violate the KSS bound, as it happens in other anisotropic models $[56,57]$. As a preliminary calculation one can consider the perturbation of the $x^{1} x^{3}$ component of the metric and study the response function. By using the standard Kubo formalism in the holographic setup, we get (see, for example, [29]):

$$
\frac{\eta_{\perp}}{s}=\left.\frac{g_{x^{1} x^{1}}}{g_{x^{3} x^{3}}}\right|_{r=r_{h}} .
$$

The value of the transverse viscosity $\eta_{\perp}$ obtained in this way satisfies $\eta_{\perp} / s \sim Q_{c}^{\frac{2}{3}} Q_{f}^{-\frac{4}{3}} T^{\frac{4}{3}}$, which certainly can be arbitrary small as $T \rightarrow 0$ and, therefore, violates the KSS bound at low temperatures. 
One important feature of our geometry is that it does not have a weak anisotropy limit and, in fact, it is non-analytic in $Q_{f}$ when $Q_{f} \rightarrow 0$. This is due to the fact that the flavors introduced are massless. It was shown in [25] how to generalize the supersymmetric $(T=0)$ solution to the case in which the flavors are massive. In this case the flavor branes do not reach the origin and there is a cavity around $r=0$ in which the D5-brane charge is zero and the equations of motion are those of the unflavored system. The radius of the cavity is related to the mass of the quarks. The massive solutions found in [25] interpolate between the unflavored metric in the IR and the massless flavored geometry in the UV. By sending the quark mass to infinity the size of the cavity increases and the geometry becomes $A d S_{5} \times \mathbb{S}^{5}$. This is quite natural from the point of view of field theory since in this infinite mass limit we are making the flavors non dynamical. From the holographic point of view, the quark mass is an external parameter which allows to modify the degree of anisotropy. It would be very interesting to generalize some of the results found here to this massive case and to explore the development of anisotropy and their effects on the physical observables. Work along these lines is in progress.

\section{Acknowledgments}

We are grateful to Y. Bea, F. Bigazzi, G. Itsios, N. Jokela, D. Musso, C. Núñez and J. Tarrío for discussions and useful suggestions. J. M. P. and A. V. R. are funded by the Spanish grant FPA2014-52218-P by Xunta de Galicia (GRC2013-024), by FEDER and by the Maria de Maeztu Unit of Excellence MDM-2016-0692. J. M. P. is supported by the Spanish FPU fellowship FPU14/06300. Centro de Física do Porto is partially funded by FCT through the project CERN/FIS-NUC/0045/2015.

\section{A Details of the background}

Let us write a coordinate representation of the internal part of our background. The metric of $\mathbb{C P}^{2}$ can be written as:

$$
d s_{\mathbb{C P}^{2}}^{2}=d \chi^{2}+\frac{\cos ^{2} \chi}{4}\left(\left(\omega^{1}\right)^{2}+\left(\omega^{2}\right)^{2}\right)+\frac{\cos ^{2} \chi \sin ^{2} \chi}{4}\left(\omega^{3}\right)^{2},
$$

where $\chi$ is an angular coordinate taking values in the range $0 \leq \chi \leq \pi$ and $\omega^{1}, \omega^{2}$ and $\omega^{3}$ are three $\mathrm{SU}(2)$ left-invariant one-forms, which can be written in terms of three angles $(\theta, \varphi, \psi)$ as follows:

$$
\begin{aligned}
& \omega^{1}=\cos \psi d \theta+\sin \psi \sin \theta d \varphi \\
& \omega^{2}=\sin \psi d \theta-\cos \psi \sin \theta d \varphi \\
& \omega^{3}=d \psi+\cos \theta d \varphi
\end{aligned}
$$

The fiber $\tau$ in (2.5) takes values in the range $0 \leq \tau \leq 2 \pi$ and the one-form $A$ is:

$$
A=\frac{1}{2} \cos ^{2}\left(\frac{\chi}{2}\right) \omega^{3} .
$$


The vielbein basis of $\mathbb{C} \mathbb{P}^{2}$ is:

$$
\begin{aligned}
e^{1} & =\frac{1}{2} \cos \left(\frac{\chi}{2}\right) \omega^{1}, & e^{2} & =\frac{1}{2} \cos \left(\frac{\chi}{2}\right) \omega^{2}, \\
e^{3} & =\frac{1}{2} \cos \left(\frac{\chi}{2}\right) \sin \left(\frac{\chi}{2}\right) \omega^{3}, & e^{4} & =\frac{1}{2} d \chi .
\end{aligned}
$$

We can use these one-forms to define the two-form $\hat{\Omega}_{2}$ as:

$$
\hat{\Omega}_{2}=e^{3 i \tau}\left(e^{1}+i e^{2}\right) \wedge\left(e^{3}+i e^{4}\right),
$$

Let us now write our ansatz for $F_{3}$ as:

$$
F_{3}=Q_{f} d x^{3} \wedge \operatorname{Im} \hat{\Omega}_{2}
$$

where $Q_{f}$ is a constant proportional to the number of flavors $N_{f}$. The modified Bianchi identity for $F_{3}$ is:

$$
d F_{3}=-3 Q_{f} d x^{3} \wedge \operatorname{Re} \hat{\Omega}_{2} \wedge(d \tau+A) .
$$

The dilaton for our solution is:

$$
e^{\frac{3 \phi}{2}}=\frac{3}{4 Q_{f}} r
$$

Moreover, the RR five-form $F_{5}$ for our background can be written as:

$$
F_{5}=\partial_{r}\left(e^{-\phi} h^{-1}\right)(1+*) d^{4} x \wedge d r .
$$

The precise relation between $Q_{f}$ and $N_{f}$ can be obtained by analyzing the embeddings of the family of flavor branes that source the background. For the case of flavor branes dual to massless quarks we get:

$$
Q_{f}=\frac{4 \pi N_{f}}{9 \sqrt{3}}
$$

\section{B Wilson loops and entanglement entropies}

In this appendix we calculate the potential energy for static quark-antiquark pairs, as well as the entanglement entropy for slab regions and their complements.

\section{B.1 Quark-antiquark potentials}

To calculate the potential energy between a "quark" and an "antiquark" we will follow the holographic prescription to compute the Wilson loops developed in $[36,37]$. In this method one has to solve the equations of motion of a fundamental string with its two ends lying at the UV boundary. These equations are obtained by extremizing the Nambu-Goto action:

$$
S=\frac{1}{2 \pi} \int d \tau d \sigma e^{\frac{\phi}{2}} \sqrt{-\operatorname{det} g_{2}},
$$

where $g_{2}$ is the Einstein frame induced metric on the worldvolume of the string. We consider separately the cases in which the quark and the antiquark are in the same layer (i.e., with the same value of the coordinate $x^{3}$ ) and the configuration in which they have the same value of $\left(x^{1}, x^{2}\right)$ and different values of $x^{3}$. 


\section{B.1.1 Intra-layer potential}

Let us first consider a fundamental string hanging from the UV boundary $r \rightarrow \infty$ and extended along one of the layer directions (say along $x^{1} \equiv x$ ). with the other two cartesian coordinates being constant. We parametrize the worldvolume of such a string by means of the coordinates $(\tau, \sigma)=\left(x^{0}, x^{1}\right)$. The Nambu-Goto action (B.1) takes the form:

$$
\frac{S}{T}=\int d x e^{\frac{\phi}{2}} \sqrt{\left(r^{\prime}\right)^{2}+\frac{r^{4}}{R^{4}}} \equiv \int d x L
$$

where the prime denotes derivative with respect to $x, T=\int d x^{0}$ and we have defined an effective lagrangian function $L$. Since $L$ does not depend explicitly on $x$, the Euler-Lagrange equation of motion has the following first integral:

$$
r^{\prime} \frac{\partial L}{\partial r^{\prime}}-L=\text { constant }
$$

or, more explicitly:

$$
\frac{r^{4} e^{\frac{\phi}{2}}}{\sqrt{\left(r^{\prime}\right)^{2}+\frac{r^{4}}{R^{4}}}}=r_{0}^{2} R^{2} e^{\frac{\phi_{o}}{2}},
$$

where $r_{0}$ is the turning point, i.e., the minimal value of the coordinate $r$, and $\phi_{0}=\phi\left(r=r_{0}\right)$. It is now straightforward to use (B.4) to obtain $r^{\prime}$ :

$$
r^{\prime}= \pm \frac{r^{2}}{R^{2}} \sqrt{\left(\frac{r}{r_{0}}\right)^{4} e^{\phi-\phi_{0}}-1}
$$

from which we easily get the parallel cartesian coordinate $x$ as a function of the holographic coordinate $r$ :

$$
x(r)= \pm \frac{R^{2}}{r_{0}} \int_{1}^{\frac{r}{r_{0}}} \frac{d y}{y^{2} \sqrt{y^{\frac{14}{3}}-1}} .
$$

It follows that the quark-antiquark distance $d_{\|}$at the boundary is:

$$
d_{\|}=\frac{2 R^{2}}{r_{0}} \int_{1}^{\infty} \frac{d y}{y^{2} \sqrt{y^{\frac{14}{3}}-1}}=\frac{2 R^{2} \sqrt{\pi}}{r_{0}} \frac{\Gamma\left(\frac{5}{7}\right)}{\Gamma\left(\frac{3}{14}\right)} .
$$

Let us now use (B.4) to compute the on-shell action for this configuration of the fundamental string. After some calculation we get:

$$
\frac{S_{\text {on-shell }}}{T}=2 \frac{1}{2 \pi} \frac{e^{\frac{\phi_{0}}{2}}}{r_{0}^{2}} \int_{r_{0}}^{r_{\max }} \frac{r^{2} e^{\phi-\phi_{0}} d r}{\sqrt{\left(\frac{r}{r_{0}}\right)^{4} e^{\phi-\phi_{0}}-1}} .
$$

Using the value of the dilaton for our background, we obtain:

$$
\frac{S_{\text {on-shell }}}{T}=\frac{1}{\pi}\left(\frac{3}{4 Q_{f}}\right)^{\frac{1}{3}} r_{0}^{\frac{4}{3}} \int_{1}^{\frac{r_{\max }}{r_{0}}} \frac{y^{\frac{8}{3}} d y}{\sqrt{y^{\frac{14}{3}}-1}},
$$


which is a divergent integral when $r_{\max } \rightarrow \infty$. We regularize this divergence by subtracting the action of two fundamental strings going straight from $r=0$ to the boundary at $r=$ $r_{\text {rmax }}$. The resulting finite action divided by $T$ is identified with the $q \bar{q}$ potential:

$$
V_{q \bar{q}}=\frac{S_{\text {on }- \text { shell }}^{\text {reg }}}{T}=\frac{S_{\text {on-shell }}}{T}-\frac{2}{2 \pi} \int_{0}^{r_{\max }} d r e^{\frac{\phi}{2}}=\frac{S_{\text {on-shell }}}{T}-\frac{3}{4 \pi}\left(\frac{3}{4 Q_{f}}\right)^{\frac{1}{3}} r_{\max }^{\frac{4}{3}} .
$$

One can easily show that $V_{q \bar{q}}$ can be rewritten as:

$$
V_{q \bar{q}}=-\frac{1}{\pi}\left(\frac{3}{4 Q_{f}}\right)^{\frac{1}{3}} r_{0}^{\frac{4}{3}}\left[\frac{3}{4}-\int_{1}^{\infty} d y y^{\frac{1}{3}}\left(\frac{y^{\frac{7}{3}}}{\sqrt{y^{\frac{14}{3}}-1}}-1\right)\right] .
$$

The integral inside the brackets in this last expression can be computed analytically. We get:

$$
V_{q \bar{q}}=-\frac{3}{4 \sqrt{\pi}}\left(\frac{3}{4 Q_{f}}\right)^{\frac{1}{3}} r_{0}^{\frac{4}{3}} \frac{\Gamma\left(\frac{5}{7}\right)}{\Gamma\left(\frac{3}{14}\right)} .
$$

By using the relation (B.7) we can eliminate $r_{0}$ in favor of the $q \bar{q}$ distance $d_{\|}$. After some calculation we get:

$$
V_{q \bar{q}}=-\beta_{\|} \frac{Q_{c}^{\frac{2}{3}}}{Q_{f}^{\frac{1}{3}}} \frac{1}{d_{\|}^{\frac{4}{3}}}, \quad \quad \beta_{\|}=\frac{16 \pi^{\frac{1}{6}}}{9 \cdot 5^{\frac{2}{3}}}\left(\frac{\Gamma\left(\frac{5}{7}\right)}{\Gamma\left(\frac{3}{14}\right)}\right)^{\frac{7}{3}} .
$$

\section{B.1.2 Inter-layer potential}

Let us now repeat the analysis of the previous section for the case in which the fundamentals are separated at the boundary in the transverse direction $x^{3} \equiv z$ to the layers. We now take $\tau=x^{0}$ and $\sigma=z$ and consider an ansatz of the form $r=r(z)$. The corresponding Nambu-Goto action becomes:

$$
\frac{S}{T}=\frac{1}{2 \pi} \int d z e^{\frac{\phi}{2}} \sqrt{\left(r^{\prime}\right)^{2}+e^{-2 \phi} \frac{r^{4}}{R^{4}}} \equiv \int d z L
$$

where now $r^{\prime}=d r / d z$. Proceeding as in section B.1.1, we get:

$$
r^{\prime}=e^{-\phi} \frac{r^{2}}{R^{2}} \sqrt{\left(\frac{r}{r_{0}}\right)^{4} e^{\phi_{0}-\phi}-1}
$$

which yields the following function $z=z(r)$ :

$$
z(r)= \pm \frac{R^{2}}{r_{0}^{\frac{1}{3}}}\left(\frac{3}{4 Q_{f}}\right)^{\frac{2}{3}} \int_{1}^{\frac{r}{r_{0}}} \frac{d y}{y^{\frac{4}{3}} \sqrt{y^{\frac{10}{3}}-1}},
$$

as well as the following transverse distance:

$$
d_{\perp}=2 \frac{R^{2}}{r_{0}^{\frac{1}{3}}}\left(\frac{3}{4 Q_{f}}\right)^{\frac{2}{3}} \int_{1}^{\infty} \frac{d y}{y^{\frac{4}{3}} \sqrt{y^{\frac{10}{3}}-1}}=6 \sqrt{\pi}\left(\frac{3}{4 Q_{f}}\right)^{\frac{2}{3}} \frac{\Gamma\left(\frac{3}{5}\right)}{\Gamma\left(\frac{1}{10}\right)} \frac{R^{2}}{r_{0}^{\frac{1}{3}}} .
$$


The unregulated on-shell action in this case is given by:

$$
\frac{S_{\text {on-shell }}}{T}=\frac{e^{\frac{\phi_{0}}{2}}}{\pi r_{0}^{2}} \int_{r_{0}}^{r_{\max }} \frac{r^{2} d r}{\sqrt{\left(\frac{r}{r_{0}^{2}}\right)^{4} e^{\phi_{0}-\phi}-1}}=\frac{1}{\pi}\left(\frac{3}{4 Q_{f}}\right)^{\frac{1}{3}} r_{0}^{\frac{4}{3}} \int_{1}^{\frac{r_{\max }}{r_{0}}} \frac{y^{2} d y}{\sqrt{y^{\frac{10}{3}}-1}},
$$

whereas the $q \bar{q}$ potential is:

$$
V_{q \bar{q}}=-\frac{1}{\pi}\left(\frac{3}{4 Q_{f}}\right)^{\frac{1}{3}} r_{0}^{\frac{4}{3}}\left[\frac{3}{4}-\int_{1}^{\infty} d y y^{\frac{1}{3}}\left(\frac{y^{\frac{5}{3}}}{\sqrt{y^{\frac{10}{3}}-1}}-1\right)\right] .
$$

By performing the integral in this last expression we arrive at:

$$
V_{q \bar{q}}=-\frac{3}{4 \sqrt{\pi}}\left(\frac{3}{4 Q_{f}}\right)^{\frac{1}{3}} r_{0}^{\frac{4}{3}} \frac{\Gamma\left(\frac{3}{5}\right)}{\Gamma\left(\frac{1}{10}\right)} .
$$

Finally, we can rewrite this intra-layer potential in terms of $d_{\perp}$ as:

$$
V_{q \bar{q}}=-\beta_{\perp} \frac{Q_{c}^{2}}{Q_{f}^{3}} \frac{1}{d_{\perp}^{4}}, \quad \quad \beta_{\perp}=\frac{2^{12} \pi^{\frac{3}{2}}}{3^{2} \cdot 5^{2}}\left(\frac{\Gamma\left(\frac{3}{5}\right)}{\Gamma\left(\frac{1}{10}\right)}\right)^{5}
$$

\section{B.2 Entanglement entropy}

Let $A$ be a spatial region in the gauge theory. The holographic entanglement entropy between $A$ and its complement is obtained by finding the eight-dimensional spatial surface $\Sigma$ whose boundary coincides with the boundary of $A$ and minimizes the functional [38, 39]:

$$
S_{A}=\frac{1}{4 G_{10}} \int_{\Sigma} d^{8} \xi \sqrt{\operatorname{det} g_{8}}
$$

where $G_{10}$ is the ten-dimensional Newton constant $\left(G_{10}=8 \pi^{6}\right.$ in our units) and $g_{8}$ is the induced metric on $\Sigma$ in the Einstein frame. The entanglement entropy between $A$ and its complement is given by $S_{A}$ evaluated on the minimal surface $\Sigma$. We will obtain $S_{A}$ when $A$ is a slab extended infinitely in two spatial cartesian directions and having a finite width in the third one. We will consider separately the two cases corresponding to the two possible orientations of the slab.

\section{B.2.1 Parallel slab}

Let us consider first the case in which $A$ is the region $\left\{-\frac{l_{\|}}{2} \leq x^{1} \leq \frac{l_{\|}}{2},-\infty<x^{2}, x^{3}<\right.$ $+\infty$ \}, i.e., when the slab has a finite width in the direction parallel to the layers. We will characterize the surface $\Sigma$ by a function $r=r(x)$, where $x \equiv x^{1}$. After integrating over all coordinates except $x$, we get:

$$
\frac{S_{\|}}{L_{2} L_{3}}=\frac{R^{4}}{32 \pi^{3}}\left(\frac{3}{2 \sqrt{2}}\right)^{6} \int e^{-\phi} r \sqrt{\left(r^{\prime}\right)^{2}+\frac{r^{4}}{R^{4}}} d x
$$


where $L_{2,3}=\int d x^{2,3}$ and $r^{\prime}=d r / d x$. The Euler-Lagrange equations which minimize $S_{\|}$ admit the following first integral:

$$
\frac{r^{5} e^{-\phi}}{\sqrt{\left(r^{\prime}\right)^{2}+\frac{r^{4}}{R^{4}}}}=R^{2} r_{0}^{3} e^{-\phi_{0}}
$$

where $r_{0}$ is the minimal value of $r$ and $\phi_{0}=\phi\left(r=r_{0}\right)$. It follows that $r^{\prime}$ is given by:

$$
r^{\prime}= \pm \frac{r^{2}}{R^{2}} \sqrt{\left(\frac{r}{r_{0}}\right)^{6} e^{2\left(\phi_{0}-\phi\right)}-1}= \pm \frac{r^{2}}{R^{2}} \sqrt{\left(\frac{r}{r_{0}}\right)^{\frac{14}{3}}-1}
$$

and, therefore:

$$
x(r)= \pm \frac{R^{2}}{r_{0}} \int_{1}^{\frac{r}{r_{0}}} \frac{d y}{y^{2} \sqrt{y^{\frac{14}{3}}-1}} .
$$

Then, the length $l_{\|}$in the direction parallel to the layers is:

$$
l_{\|}=\frac{2 R^{2}}{r_{0}} \int_{1}^{\infty} \frac{d y}{y^{2} \sqrt{y^{\frac{14}{3}}-1}}=\frac{2 R^{2} \sqrt{\pi}}{r_{0}} \frac{\Gamma\left(\frac{5}{7}\right)}{\Gamma\left(\frac{3}{14}\right)} .
$$

One can now evaluate the entropy for this configuration. We get:

$$
\frac{S_{\|}}{L_{2} L_{3}}=\frac{R^{4}}{16 \pi^{3}}\left(\frac{3}{2 \sqrt{2}}\right)^{6} r_{0}^{2} e^{-\phi_{0}} \int_{1}^{\frac{r_{\max }}{r_{0}}} \frac{y^{\frac{8}{3}} d y}{\sqrt{y^{\frac{14}{3}}-1}} .
$$

The integral (B.28) is divergent at the UV and has been regulated by introducing a maximal radial coordinate $r_{\max }$. The divergent part of $S_{\|}$can be obtained by computing the contribution of the upper limit to the integral (B.28) and gives:

$$
\frac{S_{\|}^{\text {div }}}{L_{2} L_{3}}=\frac{3 R^{4}}{64 \pi^{3}}\left(\frac{3}{2 \sqrt{2}}\right)^{6}\left(\frac{4 Q_{f}}{3}\right)^{\frac{2}{3}} r_{\max }^{\frac{4}{3}} .
$$

We now define $S_{\|}^{\text {finite }}$ as:

$$
\frac{S_{\|}^{\text {finite }}}{L_{2} L_{3}}=\frac{S_{\|}-S_{\|}^{\text {div }}}{L_{2} L_{3}} .
$$

One can readily demonstrate that:

$$
\frac{S_{\|}^{\text {finite }}}{L_{2} L_{3}}=-\frac{R^{4}}{16 \pi^{3}}\left(\frac{3}{2 \sqrt{2}}\right)^{6} r_{0}^{2} e^{-\phi_{0}}\left[\frac{3}{4}-\int_{1}^{\infty} d y y^{\frac{1}{3}}\left(\frac{y^{\frac{7}{3}}}{\sqrt{y^{\frac{14}{3}}-1}}-1\right)\right],
$$

which, after performing the integration, gives:

$$
\frac{S_{\|}^{\text {finite }}}{L_{2} L_{3}}=-\frac{3 \sqrt{\pi}}{64 \pi^{3}}\left(\frac{3}{2 \sqrt{2}}\right)^{6}\left(\frac{4 Q_{f}}{3}\right)^{\frac{2}{3}} \frac{\Gamma\left(\frac{5}{7}\right)}{\Gamma\left(\frac{3}{14}\right)} R^{4} r_{0}^{\frac{4}{3}} .
$$

By using the relation (B.27) between $r_{0}$ and $l_{\|}$, we can rewrite $S_{\|}^{\text {finite }}$ as:

$$
\frac{S_{\|}^{\text {finite }}}{L_{2} L_{3}}=-\gamma_{\|} \frac{Q_{f}^{\frac{2}{3}} Q_{c}^{\frac{5}{3}}}{l_{\|}^{\frac{4}{3}}}, \quad \gamma_{\|}=\frac{2}{45 \cdot 5^{\frac{2}{3}} \pi^{\frac{11}{6}}}\left(\frac{\Gamma\left(\frac{5}{7}\right)}{\Gamma\left(\frac{3}{14}\right)}\right)^{\frac{7}{3}} .
$$




\section{B.2.2 Transverse slab}

We now take $A$ to be $\left\{-\infty<x^{1}, x^{2}<+\infty,-\frac{l_{\perp}}{2} \leq x^{3} \leq \frac{l_{\perp}}{2}\right\}$, i.e., a slab with finite width in the direction $x^{3}$ transverse to the layers. If $z \equiv x^{3}$, the surface $\Sigma$ is parametrized by a function $r=r(z)$ and the functional to be minimized is:

$$
\frac{S_{\perp}}{L_{1} L_{2}}=\frac{R^{4}}{32 \pi^{3}}\left(\frac{3}{2 \sqrt{2}}\right)^{6} \int r \sqrt{\left(r^{\prime}\right)^{2}+\frac{r^{4}}{R^{4}} e^{-2 \phi}} d z .
$$

The corresponding first integral is now:

$$
\frac{r^{5} e^{-2 \phi}}{\sqrt{\left(r^{\prime}\right)^{2}+\frac{r^{4}}{R^{4}} e^{-2 \phi}}}=R^{2} r_{0}^{3} e^{-\phi_{0}},
$$

and, as a consequence, $r^{\prime}$ is given by:

$$
r^{\prime}= \pm \frac{r^{2}}{R^{2}} e^{-\phi} \sqrt{\left(\frac{r}{r_{0}}\right)^{6} e^{2\left(\phi_{0}-\phi\right)}-1}= \pm \frac{r^{2}}{R^{2}} e^{-\phi} \sqrt{\left(\frac{r}{r_{0}}\right)^{\frac{14}{3}}-1}
$$

Therefore $z(r)$ is the following integral:

$$
z(r)= \pm\left(\frac{3}{4 Q_{f}}\right)^{\frac{2}{3}} \frac{R^{2}}{r_{0}^{\frac{1}{3}}} \int_{1}^{\frac{r}{r_{0}}} \frac{d y}{y^{\frac{4}{3}} \sqrt{y^{\frac{14}{3}}-1}},
$$

and the transverse length $l_{\perp}$ is related to $r_{0}$ as:

$$
l_{\perp}=6 \sqrt{\pi}\left(\frac{3}{4 Q_{f}}\right)^{\frac{2}{3}} \frac{\Gamma\left(\frac{4}{7}\right)}{\Gamma\left(\frac{1}{14}\right)} \frac{R^{2}}{r_{0}^{\frac{1}{3}}} .
$$

The functional $S_{\perp}$ evaluated on the minimal surface is given by:

$$
\frac{S_{\perp}}{L_{1} L_{2}}=\frac{R^{4}}{16 \pi^{3}}\left(\frac{3}{2 \sqrt{2}}\right)^{6} r_{0}^{2} \int_{1}^{\frac{r_{\max }}{r_{0}}} \frac{y^{\frac{10}{3}} d y}{\sqrt{y^{\frac{14}{3}}-1}},
$$

and its divergent part is:

$$
\frac{S_{\perp}^{\text {div }}}{L_{1} L_{2}}=\frac{R^{4}}{32 \pi^{3}}\left(\frac{3}{2 \sqrt{2}}\right)^{6} r_{\max }^{2}
$$

Defining $S_{\perp}^{\text {finite }}$ by subtracting $S_{\perp}^{\text {div }}$ from $S_{\perp}$ :

$$
\frac{S_{\perp}^{\text {finite }}}{L_{1} L_{2}}=\frac{S_{\perp}-S_{\perp}^{\text {div }}}{L_{1} L_{2}}
$$

we get:

$$
\frac{S_{\perp}^{\text {finite }}}{L_{1} L_{2}}=-\frac{R^{4}}{16 \pi^{3}}\left(\frac{3}{2 \sqrt{2}}\right)^{6} r_{0}^{2}\left[\frac{1}{2}-\int_{1}^{\infty} d y y\left(\frac{y^{\frac{7}{3}}}{\sqrt{y^{\frac{14}{3}}-1}}-1\right)\right]
$$


which, after computing the integral, becomes:

$$
\frac{S_{\perp}^{\text {finite }}}{L_{1} L_{2}}=-\frac{1}{32 \pi^{3}}\left(\frac{3}{2 \sqrt{2}}\right)^{6} \sqrt{\pi} \frac{\Gamma\left(\frac{4}{7}\right)}{\Gamma\left(\frac{1}{14}\right)} R^{4} r_{0}^{2} .
$$

Finally, using the relation (B.38), we arrive at:

$$
\frac{S_{\perp}^{\text {finite }}}{L_{1} L_{2}}=-\gamma_{\perp} \frac{Q_{c}^{4}}{Q_{f}^{4}} \frac{1}{l_{\perp}^{6}}, \quad \gamma_{\perp}=\left(\frac{16}{15}\right)^{4} \sqrt{\pi}\left(\frac{\Gamma\left(\frac{4}{7}\right)}{\Gamma\left(\frac{1}{14}\right)}\right)^{7}
$$

\section{More on the reduced equations}

In this appendix we give details on the dimensional reduction of our setup. We first consider the reduction to four dimensions.

\section{C.1 4d reduction}

Let us consider the reduction ansatz of the $10 \mathrm{~d}$ metric written in (4.1). For this ansatz, the determinant of the $10 \mathrm{~d}$ and $4 \mathrm{~d}$ metrics are related as:

$$
\sqrt{-G_{10}}=e^{\frac{10}{3} \gamma-\beta} \sqrt{G_{5}} \sqrt{-g_{4}}
$$

where $G_{5}$ is the determinant of the $5 \mathrm{~d}$ compact internal manifold. Moreover, the relation between the Ricci scalars in $10 \mathrm{~d}$ and $4 \mathrm{~d}$ is:

$R_{10}=e^{-\frac{10}{3} \gamma+\beta}\left[R_{4}-\frac{40}{3}(\partial \gamma)^{2}-20(\partial \lambda)^{2}-\frac{3}{2}(\partial \beta)^{2}+24 e^{\frac{16}{3} \gamma+2 \lambda-\beta}-4 e^{\frac{16}{3} \gamma+12 \lambda-\beta}+\Lambda\right]$,

where $\Lambda$ is given by:

$$
\Lambda=\frac{1}{\sqrt{-g_{4}}} \partial_{m}\left[\sqrt{-g_{4}} g^{m n} \partial_{n}\left(\beta-\frac{10}{3} \gamma\right)\right] .
$$

As $\Lambda$ leads to a total derivative in the $4 \mathrm{~d}$ Einstein-Hilbert action and, thus, it does not contribute to the equations of motion and we simply drop it from our equations. The Einstein-Hilbert action in 10d can be written as:

$$
\begin{aligned}
\int d^{10} X \sqrt{-G_{10}} R_{10}=V_{5} V_{x^{3}} \int d^{4} z \sqrt{-g_{4}}[ & R_{4}-\frac{40}{3}(\partial \gamma)^{2}-20(\partial \lambda)^{2}-\frac{3}{2}(\partial \beta)^{2}+ \\
& \left.+24 e^{\frac{16}{3} \gamma+2 \lambda-\beta}-4 e^{\frac{16}{3} \gamma+12 \lambda-\beta}\right], \quad(\text { C. })
\end{aligned}
$$

where $V_{5}$ is the volume of the five-dimensional compact space and $V_{x^{3}} \equiv \int d x^{3}$.

Let us now write the contribution of the remaining fields of type IIB supergravity to the reduced action. We start with the contribution of the dilaton $\phi$, which is proportional to:

$$
\int d^{10} X \sqrt{-G_{10}} \frac{1}{2} G^{M N} \partial_{M} \phi \partial_{N} \phi=V_{5} V_{x^{3}} \int d^{4} z \sqrt{-g_{4}} \frac{1}{2} g^{m n} \partial_{m} \phi \partial_{n} \phi .
$$


Moreover, the RR five-form $F_{5}$ in these new variables is:

$$
F_{5}=Q_{c} e^{\frac{40}{3} \gamma-\beta} \sqrt{-g_{4}} d^{4} z \wedge d x^{3}
$$

and its contribution to the effective action is proportional to:

$$
\int \frac{1}{2} F_{5} \wedge^{*} F_{5}=V_{5} V_{x^{3}} \int d^{4} z \sqrt{-g_{4}} \frac{Q_{c}^{2}}{2} e^{\frac{40}{3} \gamma-\beta}
$$

Similarly, the RR three-form $F_{3}$ contributes as:

$$
\int \frac{1}{2} e^{\phi} F_{3} \wedge^{*} F_{3}=V_{5} V_{x^{3}} \int d^{4} z \sqrt{-g_{4}} Q_{f}^{2} e^{\phi+4 \gamma+4 \lambda-3 \beta} .
$$

It remains to calculate the contribution of the DBI action of the flavor D5-branes, which is given by:

$$
-\frac{3 V_{5} V_{x^{3}} Q_{f}}{\kappa_{10}^{2}} \int d^{4} z \sqrt{-g_{4}} e^{\frac{14}{3} \gamma-2 \beta-2 \lambda+\frac{\phi}{2}}
$$

Putting everything together, we can write the effective action as in (4.2), where $V$ is the potential for the scalar fields $\phi, \gamma, \lambda$ and $\beta$ written in (4.3).

Let us now write down the equations of motion derived from the action (4.2). First of all, the equation of motion for the $4 \mathrm{~d}$ metric is:

$$
R_{m n}=\frac{1}{2} \partial_{m} \phi \partial_{n} \phi+\frac{40}{3} \partial_{m} \gamma \partial_{n} \gamma+20 \partial_{m} \lambda \partial_{n} \lambda+\frac{3}{2} \partial_{m} \beta \partial_{n} \beta+\frac{1}{2} g_{m n} V,
$$

where $R_{m n}$ is the Ricci tensor for $g_{m n}$. These equations are equivalent to the ones written in (4.6). Moreover, if we define the d'Alembertian of any scalar field $\Psi$ as in (4.7), the equations for $\phi, \gamma, \lambda$ and $\beta$ are:

$$
\begin{aligned}
\square \phi & =\partial_{\phi} V, & \square \gamma & =\frac{3}{80} \partial_{\gamma} V, \\
\square \lambda & =\frac{1}{40} \partial_{\lambda} V, & \square \beta & =\frac{1}{3} \partial_{\beta} V,
\end{aligned}
$$

where we have denoted $\frac{\partial V}{\partial \phi}=\partial_{\phi} V$ and similarly for the other scalar fields. Notice that the four equations in (C.11) can be written more compactly as in (4.8). Let us now write the equations (C.11) for the scalars more explicitly:

$$
\begin{aligned}
& \square \phi=Q_{f}^{2} e^{4 \gamma+4 \lambda-3 \beta+\phi}+3 Q_{f} e^{\frac{14}{3} \gamma-2 \lambda-2 \beta+\frac{\phi}{2}}, \\
& \square \gamma=-\frac{24}{5} e^{\frac{16}{3} \gamma+2 \lambda-\beta}+\frac{4}{5} e^{\frac{16}{3} \gamma+12 \lambda-\beta}+\frac{3 Q_{f}^{2}}{20} e^{4 \gamma+4 \lambda-3 \beta+\phi}+\frac{Q_{c}^{2}}{4} e^{\frac{40}{3} \gamma-\beta}+\frac{21 Q_{f}}{20} e^{\frac{14}{3} \gamma-2 \lambda-2 \beta+\frac{\phi}{2}}, \\
& \square \lambda=-\frac{6}{5} e^{\frac{16}{3} \gamma+2 \lambda-\beta}+\frac{6}{5} e^{\frac{16}{3} \gamma+12 \lambda-\beta}+\frac{Q_{f}^{2}}{10} e^{4 \gamma+4 \lambda-3 \beta+\phi}-\frac{3 Q_{f}}{10} e^{\frac{14}{3} \gamma-2 \lambda-2 \beta+\frac{\phi}{2}}, \\
& \square \beta=8 e^{\frac{16}{3} \gamma+2 \lambda-\beta}-\frac{4}{3} e^{\frac{16}{3} \gamma+12 \lambda-\beta}-Q_{f}^{2} e^{4 \gamma+4 \lambda-3 \beta+\phi}-\frac{Q_{c}^{2}}{6} e^{\frac{40}{3} \gamma-\beta}-4 Q_{f} e^{\frac{14}{3} \gamma-2 \lambda-2 \beta+\frac{\phi}{2}} .
\end{aligned}
$$




\section{C.2 5d reduction}

Let us now consider a reduction of the $10 \mathrm{~d}$ metric to a $5 \mathrm{~d}$ metric according to the ansatz (5.1). The determinants of the $10 \mathrm{~d}$ and $5 \mathrm{~d}$ metrics are related as:

$$
\sqrt{-G_{10}}=e^{\frac{10}{3} \gamma} \sqrt{G_{5}} \sqrt{-g_{5}}
$$

where $G_{5}$ is the determinant of the $5 \mathrm{~d}$ compact internal manifold. Up to terms which give a total derivative in the Einstein-Hilbert action, the Ricci scalars in 10d and 5d are related as:

$$
R_{10}=e^{-\frac{10}{3} \gamma}\left[R_{5}-\frac{40}{3}(\partial \gamma)^{2}-20(\partial \lambda)^{2}+24 e^{\frac{16}{3} \gamma+2 \lambda}-4 e^{\frac{16}{3} \gamma+12 \lambda}\right] .
$$

Then, the Einstein-Hilbert action in 10d can be written as:

$$
\int d^{10} X \sqrt{-G_{10}} R_{10}=V_{5} \int d^{5} z \sqrt{-g_{5}}\left[R_{5}-\frac{40}{3}(\partial \gamma)^{2}-20(\partial \lambda)^{2}+24 e^{\frac{16}{3} \gamma+2 \lambda}-4 e^{\frac{16}{3} \gamma+12 \lambda}\right],
$$

where $V_{5}$ is the volume of the $5 \mathrm{~d}$ compact space. Let us write the contribution of the remaining fields of type IIB supergravity to the effective action. The dilaton contributes as:

$$
\int d^{10} X \sqrt{-G_{10}} \frac{1}{2} G^{M N} \partial_{M} \phi \partial_{N} \phi=V_{5} \int d^{5} z \sqrt{-g_{5}} \frac{1}{2} g^{p q} \partial_{p} \phi \partial_{q} \phi .
$$

The RR five-form is:

$$
F_{5}=Q_{c} e^{\frac{40}{3} \gamma} \sqrt{-g_{5}} d^{5} z,
$$

and contributes to the effective action as:

$$
\int \frac{1}{2} F_{5} \wedge^{*} F_{5}=V_{5} \int d^{5} z \sqrt{-g_{5}} \frac{Q_{c}^{2}}{2} e^{\frac{40}{3} \gamma} .
$$

Let us consider the following ansatz for the RR three-form $F_{3}$ :

$$
F_{3}=\frac{1}{\sqrt{2}} \mathcal{F}_{1} \wedge \operatorname{Im} \hat{\Omega}_{2},
$$

where $\hat{\Omega}_{2}$ is the two-form (A.5) and $\mathcal{F}_{1}$ has only components along the 5 d space. We will represent $\mathcal{F}_{1}$ in terms of a scalar potential $\mathcal{V}$ as in (5.2). Then, the contribution of $\mathcal{V}$ to the action is:

$$
\frac{1}{2} \int_{\mathcal{M}_{10}} F_{3} \wedge^{*} F_{3}=\frac{V_{5}}{2} \int d^{5} z \sqrt{-g_{5}} e^{4 \gamma+4 \lambda+\phi}(\partial \mathcal{V})^{2} .
$$

The DBI action of the flavor D5-branes is:

$$
S_{D B I}=-T_{5} \sum_{N_{f}} \int d^{6} \xi e^{\frac{\phi}{2}} \sqrt{-\hat{g}_{6}}
$$

After smearing and integration over the internal manifold, the DBI action becomes:

$$
S_{D B I}=-\frac{6 Q_{f} V_{5}}{2 \kappa_{10}^{2}} \int d^{5} z \sqrt{-\hat{g}_{4}} e^{\frac{\phi}{2}+\frac{14}{3} \gamma-2 \lambda},
$$


where $\hat{g}_{4}$ is the determinant of the metric obtained by taking the pullback of the $5 \mathrm{~d}$ metric on a surface with constant $x^{3}$. Putting everything together we arrive at the effective action (5.3). The equations of motion for the scalars $\phi, \gamma$ and $\lambda$ derived from (5.3) are:

$$
\begin{aligned}
& \square \phi=\partial_{\phi} U+\frac{1}{2} e^{4 \lambda+4 \gamma+\phi}(\partial \mathcal{V})^{2}+3 Q_{f} \frac{\sqrt{-\hat{g}_{4}}}{\sqrt{-g_{5}}} e^{\frac{14}{3} \gamma-2 \lambda+\frac{\phi}{2}}, \\
& \square \gamma=\frac{3}{80} \partial_{\gamma} U+\frac{3}{40} e^{4 \lambda+4 \gamma+\phi}(\partial \mathcal{V})^{2}+\frac{21}{20} Q_{f} \frac{\sqrt{-\hat{g}_{4}}}{\sqrt{-g_{5}}} e^{\frac{14}{3} \gamma-2 \lambda+\frac{\phi}{2}}, \\
& \square \lambda=\frac{1}{40} \partial_{\lambda} U+\frac{1}{20} e^{4 \lambda+4 \gamma+\phi}(\partial \mathcal{V})^{2}-\frac{3}{10} Q_{f} \frac{\sqrt{-\hat{g}_{4}}}{\sqrt{-g_{5}}} e^{\frac{14}{3} \gamma-2 \lambda+\frac{\phi}{2}},
\end{aligned}
$$

where $\square$ is the laplacian operator for the $5 \mathrm{~d}$ metric. Let us group the $5 \mathrm{~d}$ scalars into a single three-component field $\Psi=(\phi, \gamma, \lambda)$. Then, the three scalar equations of (C.23) can be compactly written as:

$$
\square \Psi=\alpha_{\Psi} \partial_{\Psi} U+\frac{1}{2} \alpha_{\Psi}(\partial \mathcal{V})^{2} \partial_{\Psi}\left(e^{4 \lambda+4 \gamma+\phi}\right)+6 Q_{f} \frac{\sqrt{-\hat{g}_{4}}}{\sqrt{-g_{5}}} \alpha_{\Psi} \partial_{\Psi}\left(e^{\frac{14}{3} \gamma-2 \lambda+\frac{\phi}{2}}\right),
$$

where the coefficients $\alpha_{\Psi}$ are those written in (4.5) for the three scalars $(\phi, \gamma, \lambda)$. The equation of $\mathcal{V}$ is:

$$
\partial_{p}\left[\sqrt{-g_{5}} e^{4 \lambda+4 \gamma+\phi} g^{p q} \partial_{q} \mathcal{V}\right]=0,
$$

while the Einstein equations are:

$$
\begin{aligned}
R_{p q}=\sum_{\Psi} & \frac{1}{2 \alpha_{\Psi}} \partial_{p} \Psi \partial_{q} \Psi+\frac{1}{2} e^{4 \lambda+4 \gamma+\phi} \partial_{p} \mathcal{V} \partial_{q} \mathcal{V}+\frac{1}{3} g_{p q} U+ \\
& +3 Q_{f} \frac{\sqrt{-g_{4}}}{\sqrt{-g_{5}}} e^{\frac{14}{3} \gamma-2 \lambda+\frac{\phi}{2}}\left(\frac{4}{3} g_{p q}-\hat{g}_{p q}^{(4)}\right) .
\end{aligned}
$$

It is straightforward to demonstrate that the metric written in (5.5) and (5.6), together with the scalars displayed in (5.7) and (5.8), satisfy (C.23), (C.25) and (C.26).

\section{C.3 $5 \mathrm{~d} \rightarrow 4 \mathrm{~d}$ reduction}

Let us now perform an additional reduction of the $5 \mathrm{~d}$ action to four dimensions. We will reduce along the coordinate $x^{3}$ and we will adopt the following ansatz for the $5 \mathrm{~d}$ metric:

$$
d s_{5}^{2}=e^{-\beta} d s_{4}^{2}+e^{2 \beta}\left(d x^{3}\right)^{2},
$$

where $\beta$ is a new scalar which depends on the $4 \mathrm{~d}$ coordinates. The determinant of the $5 \mathrm{~d}$ metric and the one corresponding to the pullback to the surface $x^{3}=$ constant are related to the determinant $g_{4}$ of the reduced $4 \mathrm{~d}$ metric as:

$$
\sqrt{-g_{5}}=e^{-\beta} \sqrt{-g_{4}}, \quad \sqrt{-\hat{g}_{4}}=e^{-2 \beta} \sqrt{-g_{4}} .
$$

Moreover, after neglecting a total derivative, we can relate the Einstein-Hilbert term of the action (C.15) to the one corresponding to the reduced $4 \mathrm{~d}$ action as:

$$
\int d^{5} z \sqrt{-g_{5}} R_{5}=\int d^{4} z \sqrt{-g_{4}}\left(R_{4}-\frac{3}{2}(\partial \beta)^{2}\right) .
$$


Let us now split the one-form $\mathcal{F}_{1}$ as:

$$
\mathcal{F}_{1}=\chi d x^{3}+f_{1}
$$

where $f_{1}$ is a closed one-form that has legs only in the $4 \mathrm{~d}$ space. Using that:

$$
\mathcal{F}_{1}^{2}=(\partial \mathcal{V})^{2}=e^{-2 \beta} \chi^{2}+e^{\beta} f_{1}^{2},
$$

we can write the term containing $\mathcal{V}$ in (5.3) as:

$$
\sqrt{-g_{5}}\left[-\frac{1}{2} e^{4 \gamma+4 \lambda+\phi}(\partial \mathcal{V})^{2}\right]=\sqrt{-g_{4}}\left[-\frac{1}{2} e^{4 \gamma+4 \lambda+\phi}\left(e^{-3 \beta} \chi^{2}+f_{1}^{2}\right)\right] .
$$

Collecting all these results, we can write the effective action as:

$$
\begin{aligned}
S_{\mathrm{eff}}=\frac{V_{5} V_{x^{3}}}{2 \kappa_{10}^{2}} \int d^{4} z \sqrt{-g_{4}} & {\left[R_{4}-\frac{40}{3}(\partial \gamma)^{2}-20(\partial \lambda)^{2}-\frac{3}{2}(\partial \beta)^{2}-\right.} \\
& \left.-\frac{1}{2}(\partial \phi)^{2}-\frac{1}{2} e^{4 \gamma+4 \lambda+\phi} f_{1}^{2}-V\right],
\end{aligned}
$$

where the $4 \mathrm{~d}$ potential $V$ is related to the 5 d one $U$ in (5.4) by the relation:

$$
V=e^{-\beta} U+\frac{1}{2} e^{4 \gamma+4 \lambda+\phi-3 \beta} \chi^{2}+6 Q_{f} e^{\frac{14}{3} \gamma-2 \lambda-2 \beta+\frac{\phi}{2}} .
$$

It is now straightforward to verify that the action (C.33) reduces to the one written in (4.2) when we truncate the former in such a way that $f_{1}=0$ and the scalar $\chi$ takes the following constant value:

$$
\chi=\sqrt{2} Q_{f} .
$$

\section{Hydrodynamic fluctuations}

In this appendix we provide details of the analysis of the hydrodynamic fluctuations, which complement the presentation given in section 6 on the main text. We will consider separately the two channels.

\section{D.1 Shear channel}

One can show that the fluctuation equations (6.5) and (6.8) for the ansatz (6.9) reduce to:

$$
\begin{array}{r}
H_{t x}^{\prime \prime}+\partial_{r} \log \left(\frac{c_{2}^{4}}{c_{1} c_{3}}\right) H_{t x}^{\prime}+W H_{t x}-q \frac{c_{3}^{2}}{c_{2}^{2}}\left(q H_{t x}+\omega H_{x y}\right)=0, \\
H_{x y}^{\prime \prime}+\partial_{r} \log \left(\frac{c_{1} c_{2}^{2}}{c_{3}}\right) H_{x y}^{\prime}+W H_{x y}+\omega \frac{c_{3}^{2}}{c_{1}^{2}}\left(q H_{t x}+\omega H_{x y}\right)=0, \\
q c_{1}^{2} H_{x y}^{\prime}+\omega c_{2}^{2} H_{t x}^{\prime}=0,
\end{array}
$$

where $W$ is the function:

$$
W=c_{3}^{2} V+2 \partial_{r}^{2} \log c_{2}+2 \partial_{r} \log c_{2} \partial_{r} \log \left(\frac{c_{1} c_{2}^{2}}{c_{3}}\right) .
$$


One can verify easily that $W$ vanishes for our background. Therefore, we will omit it in the equations that follow in this section. Notice that the last equation in (D.1) is first-order in the radial derivative and it can be used to reduce the number of equations of the system. Actually, if we define the gauge invariant combination $X$ as in (6.10) and combine this definition and the last equation in (D.1) to express the first derivatives of $H_{t x}$ and $H_{x y}$ in terms of $X^{\prime}$, we get:

$$
H_{t x}^{\prime}=\frac{q c_{1}^{2}}{q^{2} c_{1}^{2}-\omega^{2} c_{2}^{2}} X^{\prime}, \quad H_{x y}^{\prime}=-\frac{\omega c_{2}^{2}}{q^{2} c_{1}^{2}-\omega^{2} c_{2}^{2}} X^{\prime} .
$$

Moreover, one can show that the system (D.1) reduces to the following second-order differential equation for $X$ :

$$
X^{\prime \prime}+\frac{q^{2} c_{1}^{2} \partial_{r} \log \left(\frac{c_{2}^{4}}{c_{1} c_{3}}\right)-\omega^{2} c_{2}^{2} \partial_{r} \log \left(\frac{c_{1} c_{2}^{2}}{c_{3}}\right)}{q^{2} c_{1}^{2}-\omega^{2} c_{2}^{2}} X^{\prime}-\frac{c_{3}^{2}}{c_{2}^{2} c_{1}^{2}}\left(q^{2} c_{1}^{2}-\omega^{2} c_{2}^{2}\right) X=0 .
$$

By using the values of $c_{1}, c_{2}$ and $c_{3}$ written in (4.10), one can easily demonstrate that (D.4) can be converted into (6.11).

\section{D.2 Sound channel}

Plugging (6.40) and (6.37) into (6.5) we get the following second order equation for $\hat{\Psi}(r)$ :

$$
\begin{aligned}
\hat{\Psi}^{\prime \prime}+\partial_{r} \log \left(\frac{c_{1} c_{2}^{2}}{c_{3}}\right) \hat{\Psi}^{\prime} & +\left[\frac{c_{3}^{2}}{c_{1}^{2}} \omega^{2}-\frac{c_{3}^{2}}{c_{2}^{2}} q^{2}\right] \hat{\Psi}+ \\
& +\frac{1}{2} \Psi^{\prime}\left(H_{x x}^{\prime}+H_{y y}^{\prime}-H_{t t}^{\prime}\right)=c_{3}^{2} \alpha_{\Psi} \hat{\delta}\left[\partial_{\Psi} V\right]
\end{aligned}
$$

where, for every $\Psi=(\phi, \gamma, \lambda, \beta)$, we define:

$$
\hat{\delta}\left[\partial_{\Psi} V\right]=\partial_{\phi} \partial_{\Psi} V \Phi(r)+\partial_{\gamma} \partial_{\Psi} V \Gamma(r)+\partial_{\lambda} \partial_{\Psi} V \Lambda(r)+\partial_{\beta} \partial_{\Psi} V B(r) .
$$

Let us now write the equations for the metric fluctuations, which are obtained by taking different values for the $(m, n)$ indices in (6.8). To write these equations compactly, let us denote by $\hat{\delta} V$ the following radial function:

$$
\hat{\delta} V=\partial_{\phi} V \Phi(r)+\partial_{\gamma} V \Gamma(r)+\partial_{\lambda} V \Lambda(r)+\partial_{\beta} V B(r) .
$$

Then, one can check that (6.8) is equivalent to the following second-order equations:

$$
\begin{array}{r}
H_{t t}^{\prime \prime}+\partial_{r} \log \left(\frac{c_{1}^{2} c_{2}^{2}}{c_{3}}\right) H_{t t}^{\prime}-\omega^{2} \frac{c_{3}^{2}}{c_{1}^{2}}\left(H_{x x}+H_{y y}\right)-q^{2} \frac{c_{3}^{2}}{c_{2}^{2}} H_{t t}-2 \omega q \frac{c_{3}^{2}}{c_{1}^{2}} H_{t y}-\quad \text { (D.8) } \\
-\partial_{r} \log c_{1}\left(H_{x x}^{\prime}+H_{y y}^{\prime}\right)-c_{3}^{2} \hat{\delta} V+H_{t t} \tilde{W}=0, \\
H_{t y}^{\prime \prime}+\partial_{r} \log \left(\frac{c_{2}^{4}}{c_{1} c_{3}}\right) H_{t y}^{\prime}+\omega q \frac{c_{3}^{2}}{c_{2}^{2}} H_{x x}+H_{t y} W=0, \\
H_{x x}^{\prime \prime}+\partial_{r} \log \left(\frac{c_{1} c_{2}^{3}}{c_{3}}\right) H_{x x}^{\prime}+\left(\omega^{2} \frac{c_{3}^{2}}{c_{1}^{2}}-q^{2} \frac{c_{3}^{2}}{c_{2}^{2}}\right) H_{x x}-\partial_{r} \log c_{2}\left(H_{t t}^{\prime}-H_{y y}^{\prime}\right)+c_{3}^{2} \hat{\delta} V+H_{x x} W=0, \\
H_{y y}^{\prime \prime}+\partial_{r} \log \left(\frac{c_{1} c_{2}^{3}}{c_{3}}\right) H_{y y}^{\prime}+\frac{c_{3}^{2}}{c_{1}^{2}}\left(\omega^{2} H_{y y}+2 q \omega H_{t y}\right)+q^{2} \frac{c_{3}^{2}}{c_{2}^{2}}\left(H_{t t}-H_{x x}\right)+ \\
+\partial_{r} \log c_{2}\left(H_{x x}^{\prime}-H_{t t}^{\prime}\right)+c_{3}^{2} \hat{\delta} V+H_{y y} W=0,
\end{array}
$$


together with three first order constraints associated to the gauge fixing condition (6.2):

$$
\begin{aligned}
q H_{t y}^{\prime}+\omega\left(H_{x x}^{\prime}+H_{y y}^{\prime}\right)= & \partial_{r} \log \frac{c_{1}}{c_{2}}\left(2 q H_{t y}+\omega\left(H_{x x}+H_{y y}\right)\right)-\omega \sum_{\Psi} \frac{\Psi^{\prime}}{\alpha_{\Psi}} \hat{\Psi}, \\
\omega \frac{c_{2}^{2}}{c_{1}^{2}} H_{t y}^{\prime}+q\left(H_{t t}^{\prime}-H_{x x}^{\prime}\right)= & -q \partial_{r} \log \frac{c_{1}}{c_{2}} H_{t t}+q \sum_{\Psi} \frac{\Psi^{\prime}}{\alpha_{\Psi}} \hat{\Psi}, \\
\partial_{r} \log c_{2}^{2} H_{t t}^{\prime}-\partial_{r} \log \left(c_{1} c_{2}\right)\left(H_{x x}^{\prime}+H_{y y}^{\prime}\right)= & \frac{c_{3}^{2}}{c_{1}^{2}}\left(\omega^{2}\left(H_{x x}+H_{y y}\right)+2 \omega q H_{t y}\right)+ \\
& +q^{2} \frac{c_{3}^{2}}{c_{2}^{2}}\left(H_{t t}-H_{x x}\right)+c_{3}^{2} \hat{\delta} V-\sum_{\Psi} \frac{\Psi^{\prime}}{\alpha_{\Psi}} \hat{\Psi} .
\end{aligned}
$$

In (D.8) $W$ is the function defined in (D.2), which vanishes in our background and, therefore, will be omitted from now on. The function $\tilde{W}$ appearing in the first equation in (D.8) is defined as:

$$
\tilde{W}=c_{3}^{2} V+2 \partial_{r}^{2} \log c_{1}+2 \partial_{r} \log c_{1} \partial_{r} \log \left(\frac{c_{1} c_{2}^{2}}{c_{3}}\right) .
$$

This function also vanishes in our background and will also be omitted in the equations that follow.

We now write the equations for the scalar fluctuations in terms of the new fields $Z_{\hat{\Psi}}$ defined in (6.41). With this aim, let us define $\mathcal{W}_{\phi}, \mathcal{W}_{\gamma}, \mathcal{W}_{\lambda}$ and $\mathcal{W}_{\beta}$ as the following linear combinations of the $Z_{\hat{\Psi}}$ 's:

$$
\mathcal{W}_{\Psi}=\alpha_{\Psi} \sum_{\Psi^{\prime}} \frac{\partial^{2} V}{\partial \Psi \partial \Psi^{\prime}} Z_{\hat{\Psi}^{\prime}}
$$

It turns out that the equations of motion of the scalar fluctuations can be written as:

$$
\begin{array}{r}
Z_{\Phi}^{\prime \prime}+\partial_{r} \log \left(\frac{c_{1} c_{2}^{2}}{c_{3}}\right) Z_{\Phi}^{\prime}+c_{3}^{2}\left(\frac{\omega^{2}}{c_{1}^{2}}-\frac{q^{2}}{c_{2}^{2}}\right) Z_{\Phi}-\frac{3 c_{3}^{2}}{7}\left(\mathcal{W}_{\phi}-2 \mathcal{W}_{\beta}\right)=0 \\
Z_{\Gamma}^{\prime \prime}+\partial_{r} \log \left(\frac{c_{1} c_{2}^{2}}{c_{3}}\right) Z_{\Gamma}^{\prime}+c_{3}^{2}\left(\frac{\omega^{2}}{c_{1}^{2}}-\frac{q^{2}}{c_{2}^{2}}\right) Z_{\Gamma}-c_{3}^{2} \mathcal{W}_{\gamma}=0 \\
Z_{\Lambda}^{\prime \prime}+\partial_{r} \log \left(\frac{c_{1} c_{2}^{2}}{c_{3}}\right) Z_{\Lambda}^{\prime}+c_{3}^{2}\left(\frac{\omega^{2}}{c_{1}^{2}}-\frac{q^{2}}{c_{2}^{2}}\right) Z_{\Lambda}-c_{3}^{2} \mathcal{W}_{\lambda}=0 \\
Z_{B}^{\prime \prime}+\partial_{r} \log \left(\frac{c_{1} c_{2}^{2}}{c_{3}}\right) Z_{B}^{\prime}+c_{3}^{2}\left(\frac{\omega^{2}}{c_{1}^{2}}-\frac{q^{2}}{c_{2}^{2}}\right) Z_{B}-\frac{2 c_{3}^{2}}{7}\left(2 \mathcal{W}_{\beta}-\mathcal{W}_{\phi}\right)=0 .
\end{array}
$$

By combining the first and last equations in (D.12), one can immediately show that the scalar $Z_{S}$ defined in (6.42) satisfies the simple equation:

$$
Z_{S}^{\prime \prime}+\partial_{r} \log \left(\frac{c_{1} c_{2}^{2}}{c_{3}}\right) Z_{S}^{\prime}+c_{3}^{2}\left(\frac{\omega^{2}}{c_{1}^{2}}-\frac{q^{2}}{c_{2}^{2}}\right) Z_{S}=0
$$

More explicitly, this equation can be written as:

$$
Z_{S}^{\prime \prime}+\partial_{r} \log \left(r^{\frac{13}{3}} b(r)\right) Z_{S}^{\prime}+\frac{R^{4}}{r^{4} b^{2}(r)}\left(\omega^{2}-b(r) q^{2}\right) Z_{S}=0 .
$$


One can demonstrate that the equation for the gauge invariant metric fluctuation $Z_{H}$ takes the form:

$$
Z_{H}^{\prime \prime}+\mathcal{F}(r) Z_{H}^{\prime}+\mathcal{G}(r) Z_{H}+\mathcal{H}(r) Z_{S}=0,
$$

where the functions $\mathcal{F}(r), \mathcal{G}(r)$ and $\mathcal{H}(r)$ are given by:

$$
\begin{aligned}
& \mathcal{F}(r)=\partial_{r} \log \left(\frac{c_{1} c_{2}^{2}}{c_{3}}\right)-4 \partial_{r} \log \left(\frac{c_{1}}{c_{2}}\right)+\xi_{1}(r), \\
& \mathcal{G}(r)=c_{3}^{2}\left(\frac{\omega^{2}}{c_{1}^{2}}-\frac{q^{2}}{c_{2}^{2}}\right)+4\left[\partial_{r} \log \left(\frac{c_{1}}{c_{2}}\right)\right]^{2}-\partial_{r} \log \left(\frac{c_{1}}{c_{2}}\right) \xi_{1}(r), \\
& \mathcal{H}(r)=-\frac{q^{2}}{\omega^{2}} \frac{c_{1}^{2}}{c_{2}^{2}}\left[\partial_{\beta} V\left(1-\frac{\partial_{r} \log c_{1}}{\partial_{r} \log c_{2}}\right) c_{3}^{2}+6 \beta^{\prime} \xi_{2}(r)\right],
\end{aligned}
$$

with:

$$
\begin{aligned}
& \xi_{1}(r)=\frac{q^{2} \partial_{r} c_{1}^{2} \frac{\partial_{r}^{2} \log c_{2}}{\left(\partial_{r} \log c_{2}\right)^{2}}\left(1-\frac{\partial_{r}^{2} \log c_{1} \partial_{r} \log c_{2}}{\partial_{r}^{2} \log c_{2} \partial_{r} \log c_{1}}\right)+4 \omega^{2} \partial_{r} c_{2}^{2}\left(1-\frac{\partial_{r} \log c_{1}}{\partial_{r} \log c_{2}}\right)}{q^{2} c_{1}^{2}\left(\frac{\partial_{r} \log c_{1}}{\partial_{r} \log c_{2}}+1\right)-2 \omega^{2} c_{2}^{2}}, \\
& \xi_{2}(r)=\frac{\left(q^{2} c_{1}^{2}-\omega^{2} c_{2}^{2}\right) \frac{\partial_{r}^{2} \log c_{2} \partial_{r} \log c_{1}-\partial_{r}^{2} \log c_{1} \partial_{r} \log c_{2}}{\partial_{r} \log c_{2}}+2 \omega^{2} c_{2}^{2}\left(\partial_{r} \log c_{1}-\partial_{r} \log c_{2}\right)^{2}}{q^{2} c_{1}^{2} \partial_{r} \log \left(c_{1} c_{2}\right)-2 \omega^{2} c_{2}^{2} \partial_{r} \log c_{2}} .
\end{aligned}
$$

Notice that $Z_{H}$ only couples to the scalar field $Z_{S}$. Thus, we are left with (D.13) and (D.15) to be solved in the hydrodynamic approximation.

The scalar fluctuation equation (D.14) only involves the function $Z_{S}$ and, therefore, can be studied independently of $Z_{H}$. Let us adopt the following ansatz for $Z_{S}(r)$ :

$$
Z_{S}(r)=[b(r)]^{-\frac{i \omega}{2}} K(r),
$$

for which the infalling boundary conditions at the horizon are satisfied if $K(r)$ is regular at the horizon. Actually, it is much more convenient to change variables and work in the variable $x$ defined in (6.12). Recall that the horizon is located at $x=0$, whereas the boundary is at $x=1$. The fluctuation equation (D.14) is equivalent to the following equation for $K(x)$ :

$$
K^{\prime \prime}+\frac{1-2 i \hat{\omega}}{x} K^{\prime}+\frac{\left[1-\left(1-x^{2}\right)^{\frac{7}{5}}\right] \hat{\omega}^{2}-x^{2} \hat{q}^{2}}{x^{2}\left(1-x^{2}\right)^{\frac{7}{5}}} K=0,
$$

where now the primes denote derivatives with respect to the new variable $x$. We want to solve (D.19) for low $\hat{q}$. Accordingly, we expand $K(x)$ as:

$$
K(x)=K_{0}(x)+i \hat{q} K_{1}(x)+\hat{q}^{2} K_{2}(x) .
$$

Plugging (D.20) and (6.46) into (D.19) and separating the different orders in $\hat{q}$, we find the following systems of equations:

$$
\begin{aligned}
K_{0}^{\prime \prime}+\frac{K_{0}^{\prime}}{x} & =0 \\
K_{1}^{\prime \prime}+\frac{K_{1}^{\prime}}{x} & =\frac{2 v_{s}}{x} K_{0}^{\prime}, \\
K_{2}^{\prime \prime}+\frac{K_{2}^{\prime}}{x} & =\frac{2 \Gamma}{x} K_{0}^{\prime}+\frac{1}{\left(1-x^{2}\right)^{\frac{7}{5}}}\left(1-\frac{v_{s}^{2}}{x^{2}}\right) K_{0}+\frac{v_{s}^{2}}{x^{2}} K_{0}-\frac{2 v_{s}}{x} K_{1}^{\prime} .
\end{aligned}
$$


The equation for $K_{0}(x)$ can be straightforwardly integrated in general:

$$
K_{0}(x)=c_{1}+c_{2} \log x
$$

where $c_{1}$ and $c_{2}$ are constants. The regularity requirement of $K_{0}(x)$ at $x=0$ implies that $c_{2}=0$, while the condition $K_{0}(x=1)=0$ imposes that $c_{1}$ vanishes and, thus $K_{0}(x)=0$. For this value of $K_{0}(x)$ the equation for $K_{1}(x)$ in (D.21) is the same as the one for $K_{0}(x)$. Therefore, the only valid solution for our boundary conditions is $K_{1}(x)=0$. Furthermore, the same happens for $K_{2}(x)$ and, thus, we finally have that the solution for $K(x)$ satisfying the boundary conditions is the trivial one, namely:

$$
K(x)=0 .
$$

Therefore, it follows that $Z_{S}(r)=0$, as claimed in the main text.

Open Access. This article is distributed under the terms of the Creative Commons Attribution License (CC-BY 4.0), which permits any use, distribution and reproduction in any medium, provided the original author(s) and source are credited.

\section{References}

[1] J.M. Maldacena, The large- $N$ limit of superconformal field theories and supergravity, Int. J. Theor. Phys. 38 (1999) 1113 [hep-th/9711200] [INSPIRE].

[2] J. Casalderrey-Solana, H. Liu, D. Mateos, K. Rajagopal and U.A. Wiedemann, Gauge/string duality, hot QCD and heavy ion collisions, arXiv:1101.0618 [INSPIRE].

[3] J. McGreevy, Holographic duality with a view toward many-body physics, Adv. High Energy Phys. 2010 (2010) 723105 [arXiv:0909.0518] [InSPIRE].

[4] A.V. Ramallo, Introduction to the AdS/CFT correspondence, Springer Proc. Phys. 161 (2015) 411 [arXiv:1310.4319] [INSPIRE].

[5] A. Karch and E. Katz, Adding flavor to AdS/CFT, JHEP 06 (2002) 043 [hep-th/0205236] [INSPIRE].

[6] O. DeWolfe, D.Z. Freedman and H. Ooguri, Holography and defect conformal field theories, Phys. Rev. D 66 (2002) 025009 [hep-th/0111135] [INSPIRE].

[7] J. Erdmenger, Z. Guralnik and I. Kirsch, Four-dimensional superconformal theories with interacting boundaries or defects, Phys. Rev. D 66 (2002) 025020 [hep-th/0203020] [INSPIRE].

[8] K. Skenderis and M. Taylor, Branes in AdS and pp wave space-times, JHEP 06 (2002) 025 [hep-th/0204054] [INSPIRE].

[9] D. Arean and A.V. Ramallo, Open string modes at brane intersections, JHEP 04 (2006) 037 [hep-th/0602174] [INSPIRE].

[10] V.G. Filev, C.V. Johnson and J.P. Shock, Universal holographic chiral dynamics in an external magnetic field, JHEP 08 (2009) 013 [arXiv:0903.5345] [INSPIRE].

[11] K. Jensen, A. Karch, D.T. Son and E.G. Thompson, Holographic Berezinskii-Kosterlitz-Thouless transitions, Phys. Rev. Lett. 105 (2010) 041601 [arXiv: 1002.3159] [INSPIRE]. 
[12] N. Evans, A. Gebauer, K.-Y. Kim and M. Magou, Phase diagram of the D3/D5 system in a magnetic field and a BKT transition, Phys. Lett. B 698 (2011) 91 [arXiv:1003.2694] [INSPIRE].

[13] C. Kristjansen and G.W. Semenoff, Giant D5 brane holographic Hall state, JHEP 06 (2013) 048 [arXiv:1212.5609] [INSPIRE].

[14] C. Kristjansen, R. Pourhasan and G.W. Semenoff, A holographic quantum Hall ferromagnet, JHEP 02 (2014) 097 [arXiv: 1311.6999] [INSPIRE].

[15] N. Evans and P. Jones, Holographic graphene in a cavity, Phys. Rev. D 90 (2014) 086008 [arXiv: 1407.3097] [INSPIRE].

[16] J. Gomis and C. Romelsberger, Bubbling defect CFT's, JHEP 08 (2006) 050 [hep-th/0604155] [INSPIRE].

[17] F. Bigazzi, R. Casero, A.L. Cotrone, E. Kiritsis and A. Paredes, Non-critical holography and four-dimensional CFT's with fundamentals, JHEP 10 (2005) 012 [hep-th/0505140] [INSPIRE].

[18] G. Veneziano, Some aspects of a unified approach to gauge, dual and Gribov theories, Nucl. Phys. B 117 (1976) 519 [inSPIRE].

[19] C. Núñez, A. Paredes and A.V. Ramallo, Unquenched flavor in the gauge/gravity correspondence, Adv. High Energy Phys. 2010 (2010) 196714 [arXiv:1002.1088] [InSPIRE].

[20] F. Bigazzi, A.L. Cotrone, J. Mas, A. Paredes, A.V. Ramallo and J. Tarrio, D3-D7 quark-gluon plasmas, JHEP 11 (2009) 117 [arXiv:0909.2865] [INSPIRE].

[21] N. Jokela, J. Mas, A.V. Ramallo and D. Zoakos, Thermodynamics of the brane in Chern-Simons matter theories with flavor, JHEP 02 (2013) 144 [arXiv:1211.0630] [INSPIRE].

[22] F. Bigazzi, A.L. Cotrone, J. Mas, D. Mayerson and J. Tarrio, D3-D7 quark-gluon plasmas at finite baryon density, JHEP 04 (2011) 060 [arXiv:1101.3560] [INSPIRE].

[23] A.F. Faedo, A. Kundu, D. Mateos, C. Pantelidou and J. Tarrío, Three-dimensional super Yang-Mills with compressible quark matter, JHEP 03 (2016) 154 [arXiv:1511.05484] [INSPIRE].

[24] A.F. Faedo, D. Mateos, C. Pantelidou and J. Tarrio, Towards a holographic quark matter crystal, JHEP 10 (2017) 139 [arXiv:1707.06989] [INSPIRE].

[25] E. Conde, H. Lin, J.M. Penin, A.V. Ramallo and D. Zoakos, D3-D5 theories with unquenched flavors, Nucl. Phys. B 914 (2017) 599 [arXiv:1607.04998] [INSPIRE].

[26] T. Azeyanagi, W. Li and T. Takayanagi, On string theory duals of Lifshitz-like fixed points, JHEP 06 (2009) 084 [arXiv:0905.0688] [INSPIRE].

[27] D. Mateos and D. Trancanelli, Thermodynamics and instabilities of a strongly coupled anisotropic plasma, JHEP 07 (2011) 054 [arXiv:1106.1637] [INSPIRE].

[28] M. Ammon, V.G. Filev, J. Tarrio and D. Zoakos, D3/D7 quark-gluon plasma with magnetically induced anisotropy, JHEP 09 (2012) 039 [arXiv:1207.1047] [INSPIRE].

[29] S. Jain, N. Kundu, K. Sen, A. Sinha and S.P. Trivedi, A strongly coupled anisotropic fluid from dilaton driven holography, JHEP 01 (2015) 005 [arXiv: 1406.4874] [INSPIRE].

[30] L. Cheng, X.-H. Ge and S.-J. Sin, Anisotropic plasma at finite U(1) chemical potential, JHEP 07 (2014) 083 [arXiv: 1404.5027] [INSPIRE]. 
[31] E. Banks and J.P. Gauntlett, A new phase for the anisotropic $N=4$ super Yang-Mills plasma, JHEP 09 (2015) 126 [arXiv:1506.07176] [INSPIRE].

[32] D. Roychowdhury, On anisotropic black branes with Lifshitz scaling, Phys. Lett. B 759 (2016) 410 [arXiv: 1509.05229] [InSPIRE].

[33] D. Giataganas, U. Gürsoy and J.F. Pedraza, Strongly-coupled anisotropic gauge theories and holography, arXiv: 1708.05691 [INSPIRE].

[34] E. D'Hoker, J. Estes and M. Gutperle, Exact half-BPS type IIB interface solutions. I. Local solution and supersymmetric Janus, JHEP 06 (2007) 021 [arXiv:0705.0022] [INSPIRE].

[35] E. D'Hoker, J. Estes and M. Gutperle, Exact half-BPS type IIB interface solutions. II. Flux solutions and multi-Janus, JHEP 06 (2007) 022 [arXiv:0705.0024] [INSPIRE].

[36] J.M. Maldacena, Wilson loops in large-N field theories, Phys. Rev. Lett. 80 (1998) 4859 [hep-th/9803002] [INSPIRE].

[37] S.-J. Rey and J.-T. Yee, Macroscopic strings as heavy quarks in large- $N$ gauge theory and anti-de Sitter supergravity, Eur. Phys. J. C 22 (2001) 379 [hep-th/9803001] [InSPIRE].

[38] S. Ryu and T. Takayanagi, Holographic derivation of entanglement entropy from AdS/CFT, Phys. Rev. Lett. 96 (2006) 181602 [hep-th/0603001] [INSPIRE].

[39] S. Ryu and T. Takayanagi, Aspects of holographic entanglement entropy, JHEP 08 (2006) 045 [hep-th/0605073] [INSPIRE].

[40] M.M. Caldarelli, R. Emparan and B. Van Pol, Higher-dimensional rotating charged black holes, JHEP 04 (2011) 013 [arXiv: 1012.4517] [INSPIRE].

[41] N. Itzhaki, J.M. Maldacena, J. Sonnenschein and S. Yankielowicz, Supergravity and the large- $N$ limit of theories with sixteen supercharges, Phys. Rev. D 58 (1998) 046004 [hep-th/9802042] [INSPIRE].

[42] K. Jensen and A. O'Bannon, Holography, entanglement entropy and conformal field theories with boundaries or defects, Phys. Rev. D 88 (2013) 106006 [arXiv:1309.4523] [INSPIRE].

[43] V. Balasubramanian and P. Kraus, A stress tensor for anti-de Sitter gravity, Commun. Math. Phys. 208 (1999) 413 [hep-th/9902121] [INSPIRE].

[44] J. Mas and J. Tarrio, Hydrodynamics from the Dp-brane, JHEP 05 (2007) 036 [hep-th/0703093] [INSPIRE].

[45] X. Dong, S. Harrison, S. Kachru, G. Torroba and H. Wang, Aspects of holography for theories with hyperscaling violation, JHEP 06 (2012) 041 [arXiv:1201.1905] [INSPIRE].

[46] A. Batrachenko, J.T. Liu, R. McNees, W.A. Sabra and W.Y. Wen, Black hole mass and Hamilton-Jacobi counterterms, JHEP 05 (2005) 034 [hep-th/0408205] [INSPIRE].

[47] P.K. Kovtun and A.O. Starinets, Quasinormal modes and holography, Phys. Rev. D 72 (2005) 086009 [hep-th/0506184] [INSPIRE].

[48] J.I. Kapusta and T. Springer, Shear transport coefficients from gauge/gravity correspondence, Phys. Rev. D 78 (2008) 066017 [arXiv:0806.4175] [INSPIRE].

[49] P. Benincasa, A. Buchel and A.O. Starinets, Sound waves in strongly coupled non-conformal gauge theory plasma, Nucl. Phys. B 733 (2006) 160 [hep-th/0507026] [INSPIRE].

[50] P. Benincasa and A. Buchel, Hydrodynamics of Sakai-Sugimoto model in the quenched approximation, Phys. Lett. B 640 (2006) 108 [hep-th/0605076] [INSPIRE]. 
[51] A. Buchel, Relaxation time of non-conformal plasma, Phys. Lett. B 681 (2009) 200 [arXiv:0908.0108] [INSPIRE].

[52] A. Buchel, Bulk viscosity of gauge theory plasma at strong coupling, Phys. Lett. B 663 (2008) 286 [arXiv:0708.3459] [InSPIRE].

[53] T. Springer, Second order hydrodynamics for a special class of gravity duals, Phys. Rev. D 79 (2009) 086003 [arXiv:0902.2566] [InSPIRE].

[54] T. Springer, Hydrodynamics of strongly coupled non-conformal fluids from gauge/gravity duality, arXiv:0908.1587 [INSPIRE].

[55] D. Mateos, R.C. Myers and R.M. Thomson, Thermodynamics of the brane, JHEP 05 (2007) 067 [hep-th/0701132] [INSPIRE].

[56] A. Rebhan and D. Steineder, Violation of the holographic viscosity bound in a strongly coupled anisotropic plasma, Phys. Rev. Lett. 108 (2012) 021601 [arXiv:1110.6825] [INSPIRE].

[57] K.A. Mamo, Holographic RG flow of the shear viscosity to entropy density ratio in strongly coupled anisotropic plasma, JHEP 10 (2012) 070 [arXiv:1205.1797] [INSPIRE]. 